\title{
Typical Sequences Revisited - Computing Width Parameters of Graphs
}

\author{
Hans L. Bodlaender ${ }^{1}$ (D) . Lars Jaffke ${ }^{2}$ (D) . Jan Arne Telle ${ }^{2}$
}

Accepted: 7 January 2021 /Published online: 26 March 2021

(C) The Author(s) 2021

\begin{abstract}
In this work, we give a structural lemma on merges of typical sequences, a notion that was introduced in 1991 [Lagergren and Arnborg, Bodlaender and Kloks, both ICALP 1991] to obtain constructive linear time parameterized algorithms for treewidth and pathwidth. The lemma addresses a runtime bottleneck in those algorithms but so far it does not lead to asymptotically faster algorithms. However, we apply the lemma to show that the cutwidth and the modified cutwidth of series parallel digraphs can be computed in polynomial time.
\end{abstract}

Keywords Typical sequences · Treewidth - Series parallel digraphs · Cutwidth Modified cutwidth

\section{Introduction}

In this paper we revisit an old key technique from what currently are the theoretically fastest parameterized algorithms for treewidth and pathwidth, namely the use of typical sequences, and give additional structural insights for this technique. In particular, we show a structural lemma, which we call the Merge Dominator Lemma. The technique of typical sequences brings with it a partial order on sequences of integers, and a notion of possible merges of two integer sequences; surprisingly, the Merge

This article belongs to the Topical Collection: Special Issue on Theoretical Aspects of Computer Science (STACS 2020)

Guest Editors: Christophe Paul and Markus Bläser

This work was started when the third author was visiting Universitat Politecnica de Valencia, and part of it was done while the second author was visiting Utrecht University. The first author was partially supported by the Networks project, funded by the Netherlands Organization for Scientific Research (NWO). The second author is supported by the Bergen Research Foundation (BFS).

Lars Jaffke

lars.jaffke@uib.no

Extended author information available on the last page of the article. 
Dominator Lemma states that for any pair of integer sequences there exists a single merge that dominates all merges of these integer sequences, and this dominating merge can be found in linear time. On its own, this lemma does not lead to asymptotically faster parameterized algorithms for treewidth and pathwidth, but, as we discuss below, it is a concrete step towards such algorithms.

The notion of typical sequences was introduced independently in 1991 by Lagergren and Arnborg [17] and Bodlaender and Kloks [8]. In both papers, it is a key element in an explicit dynamic programming algorithm that given a tree decomposition of bounded width $\ell$, decides if the pathwidth or treewidth of the input graph $G$ is at most a constant $k$. Lagergren and Arnborg build upon this result and show that the set of forbidden minors of graphs of treewidth (or pathwidth) at most $k$ is computable; Bodlaender and Kloks show that the algorithm can also construct a tree or path decomposition of width at most $k$, if existing, in the same asymptotic time bounds. The latter result is a main subroutine in Bodlaender's linear time algorithm [3] for treewidth- $k$. If one analyses the running time of Bodlaender's algorithm for treewidth or pathwidth $\leq k$, then one can observe that the bottleneck is in the subroutine that calls the Bodlaender-Kloks dynamic programming subroutine, with both the subroutine and the main algorithm having time $\mathcal{O}\left(2^{\mathcal{O}\left(k^{3}\right)} n\right)$ for treewidth, and $\mathcal{O}\left(2^{\mathcal{O}\left(k^{2}\right)} n\right)$ for pathwidth. See also the recent work by Fürer for pathwidth [13], and the simplified versions of the algorithms of [3, 8] by Althaus and Ziegler [1]. Now, over a quarter of a century after the discovery of these results, even though much work has been done on treewidth recognition algorithms (see e.g. [2, $5,11-13,16,18,19])$, these bounds on the function of $k$ are still the best known, i.e., no $\mathcal{O}\left(2^{o\left(k^{3}\right)} n^{O(1)}\right)$ algorithm for treewidth, and no $\mathcal{O}\left(2^{o\left(k^{2}\right)} n^{O(1)}\right)$ algorithm for pathwidth is known. An interesting question, and a long-standing open problem in the field [4, Problem 2.7.1], is whether such algorithms can be obtained. Possible approaches to answer such a question is to design (e.g. ETH or SETH based) lower bounds, find an entirely new approach to compute treewidth or pathwidth in a parameterized setting, or improve upon the dynamic programming algorithms of [17] and [8]. Using our Merge Dominator Lemma we can go one step towards the latter, as follows.

The algorithms of Lagergren and Arnborg [17] and Bodlaender and Kloks [8] are based upon tabulating characteristics of tree or path decompositions of subgraphs of the input graph; a characteristic consists of an intersection model, that tells how the vertices in the current top bag interact, and for each part of the intersection model, a typical sequence of bag sizes. ${ }^{1}$ The set of characteristics for a join node is computed from the sets of characteristics of its (two) children. In particular, each pair of characteristics with one from each child can give rise to exponentially (in $k$ ) many characteristics for the join node. This is because exponentially many typical sequences may arise as the merges of the typical sequences that are part of the characteristics. In the light of our Merge Dominator Lemma, only one of these merges has

\footnotetext{
${ }^{1}$ This approach was later used in several follow up results to obtain explicit constructive parameterized algorithms for other graph width measures, like cutwidth [23, 24], branchwidth [9], different types of search numbers like linear width [10], and directed vertex separation number [7].
} 
to be stored, reducing the number of characteristics arising from each pair of characteristics of the children from $2^{\mathcal{O}(k)}$ to just 1 . Moreover, this dominating merge can be found using $\mathcal{O}(k)$ integer operations (which translates to time linear in the size of the sequence) with no large hidden constants.

Merging typical sequences at a join node is however not the only way the number of characteristics can increase throughout the algorithm, e.g. at introduce nodes, the number of characteristics increases in a different way. Nevertheless, the number of intersection models is $\mathcal{O}\left(k^{\mathcal{O}(k)}\right)$ for pathwidth and $\mathcal{O}\left(k^{\mathcal{O}\left(k^{2}\right)}\right)$ for treewidth; perhaps, with additional techniques, the number of typical sequences per part can be better bounded - in the case that a single dominating typical sequence per part suffices, this would reduce the number of table entries per node to $\mathcal{O}\left(k^{\mathcal{O}(k)}\right)$ for pathwidth- $k$, and to $\mathcal{O}\left(k^{\mathcal{O}\left(k^{2}\right)}\right)$ for treewidth- $k$, and yield $\mathcal{O}\left(k^{\mathcal{O}(k)} n\right)$ and $\mathcal{O}\left(k^{\mathcal{O}\left(k^{2}\right)} n\right)$ time algorithms for the respective problems. Concretely, suppose one could prove an analogue to the Merge Dominator Lemma for introduce nodes, stating that given a characteristic stored at their child, there is a single characteristic that dominates all the others that could potentially arise. Then the above mentioned bound of a single dominating typical sequence per part in the intersection model would follow and therefore, so would the improved algorithms for computing treewidth and pathwidth.

We give direct algorithmic consequences of the Merge Dominator Lemma in the realm of computing width parameters of directed acyclic graphs (DAGs). Specifically, we show that the (Weighted) Cutwidth and Modified CutwidTh problems on DAGs, which given a directed acyclic graph ask for the topological order that minimizes the cutwidth and modified cutwidth, respectively, ${ }^{2}$ can be solved in polynomial time on series parallel digraphs. Note that the restriction of the solution to be a topological order has been made as well in other works, e.g. [6].

Our algorithm for CUTWIDTH of series parallel digraphs has the same structure as the dynamic programming algorithm for undirected CUTWIDTH [6], but, in addition to obeying directions of edges, we have a step that only keeps characteristics that are not dominated by another characteristic in a table of characteristics. Now, with help of our Merge Dominator Lemma, we can show that in the case of series parallel digraphs, there is a unique dominating characteristic; the dynamic programming algorithm reverts to computing for each intermediate graph a single 'optimal partial solution'. This strategy also works in the presence of edge weights, which gives the algorithm for the corresponding WEIGHTED CUTWIDTH problem on series parallel digraphs. Note that the cutwidth of a directed acyclic graph is at least the maximum indegree or outdegree of a vertex; e.g., a series parallel digraph formed by the parallel composition of $n-2$ paths with three vertices has $n$ vertices and cutwidth $n-2$. To compute the modified cutwidth of a series parallel digraph, we give a linear time reduction to the WEIGHTED CUTWIDTH problem on series parallel digraphs.

\footnotetext{
${ }^{2}$ For a topological order $v_{1}, \ldots, v_{n}$ of a DAG, its cutwidth is the maximum, over all $i \in\{1, \ldots, n-1\}$, of the number of arcs whose tail is in $\left\{v_{1}, \ldots, v_{i}\right\}$ and whose head is in $\left\{v_{i+1}, \ldots, v_{n}\right\}$, while its modified cutwidth is the maximum, over all $i \in\{2, \ldots, n-1\}$, of the number of arcs whose tail is in $\left\{v_{1}, \ldots, v_{i-1}\right\}$ and whose head is in $\left\{v_{i+1}, \ldots, v_{n}\right\}$.
} 
This paper is organized as follows. In Section 2, we give a number of preliminary definitions, and review existing results, including several results on typical sequences from [8]. In Section 3, we state and prove the main technical result of this work, the Merge Dominator Lemma. Section 4 gives our algorithmic applications of this lemma, and shows that the directed cutwidth and directed modified cutwidth of a series parallel digraph can be computed in polynomial time. Some final remarks are made in Section 5.

\section{Preliminaries}

We use the following notation. For two integers $a, b \in \mathbb{Z}$ with $a \leq b$, we let $[a . . b]:=$ $\{a, a+1, \ldots, b\}$ and for $a>0$, we let $[a]:=[1 . . a]$. If $X$ is a set of size $n$, then a linear order is a bijection $\pi: X \rightarrow[n]$. Given a subset $X^{\prime} \subseteq X$ of size $n^{\prime} \leq n$, we define the restriction of $\pi$ to $X^{\prime}$ as the bijection $\left.\pi\right|_{X^{\prime}}: X^{\prime} \rightarrow\left[n^{\prime}\right]$ which is such that for all $x^{\prime}, y^{\prime} \in X^{\prime},\left.\pi\right|_{X^{\prime}}\left(x^{\prime}\right)<\left.\pi\right|_{X^{\prime}}\left(y^{\prime}\right)$ if and only if $\pi\left(x^{\prime}\right)<\pi\left(y^{\prime}\right)$.

Integer Operations In this work, the runtime of several algorithms is stated in terms of the number of required integer operations. Here, by integer operations we mean basic manipulations such as adding two integers, or comparing two integers. For integers whose absolute value is at most some $n \in \mathbb{N}$, such operations can be performed in $\mathcal{O}(\log n)$ time. Moreover, whenever we give the runtime of an algorithm in terms of the number of integer operations, then the time it takes to execute the algorithm is also upper bounded in terms of the time it takes to execute these integer operations. For instance, an algorithm that 'uses $\mathcal{O}(f(n))$ integer operations' where the value of each integer in the instance is upper bounded by $m$ runs in $\mathcal{O}(f(n) \log m)$ time.

Sequences and Matrices We denote the elements of a sequence $s$ by $s(1), \ldots, s(n)$. We denote the length of $s$ by $l(s)$, i.e., $l(s):=n$. For two sequences $a=$ $a(1), \ldots, a(m)$ and $b=b(1), \ldots, b(n)$, we denote their concatenation by $a \circ b=$ $a(1), \ldots, a(m), b(1), \ldots, b(n)$. For two sets of sequences $A$ and $B$, we let $A \odot B:=$ $\{a \circ b \mid a \in A \wedge b \in B\}$. For a sequence $s$ of length $n$ and a set $X \subseteq[n]$, we denote by $s[X]$ the subsequence of $s$ induced by $X$, i.e., let $X=\left\{x_{1}, \ldots, x_{m}\right\}$ be such that for all $i \in[m-1], x_{i}<x_{i+1}$; then, $s[X]:=s\left(x_{1}\right), \ldots, s\left(x_{m}\right)$. For $x_{1}, x_{2} \in[n]$ with $x_{1} \leq x_{2}$, we use the shorthand ' $s\left[x_{1} \ldots x_{2}\right]$ ' for ' $s\left[\left[x_{1} . . x_{2}\right]\right]$ '.

An (integer) matrix $M \in \mathbb{Z}^{m \times n}$ is said to have $m$ rows and $n$ columns. ${ }^{3}$ For sets $X \subseteq[m]$ and $Y \subseteq[n]$, we denote by $M[X, Y]$ the submatrix of $M$ induced by $X$ and $Y$, which consists of all the entries from $M$ whose indices are in $X \times Y$. For $x_{1}, x_{2} \in[m]$ with $x_{1} \leq x_{2}$ and $y_{1}, y_{2} \in[n]$ with $y_{1} \leq y_{2}$, we use the shorthand ' $M\left[x_{1} . . x_{2}, y_{1} . . y_{2}\right]$ ' for ' $M\left[\left[x_{1} . . x_{2}\right],\left[y_{1} . . y_{2}\right]\right]$ '. For a sequence $s(1), s(2), \ldots, s(\ell)$ of indices of a matrix $M$, we let

$$
M[s]:=M[s(1)], M[s(2)], \ldots, M[s(\ell)]
$$

\footnotetext{
${ }^{3}$ Since all matrices considered in this work are integer matrices, we will simply refer to them as matrices.
} 
be the corresponding sequence of entries from $M$.

For illustrative purposes we enumerate the columns of a matrix in a bottom-up fashion throughout this paper, i.e., we consider the index $(1,1)$ as the 'bottom left corner' and the index $(m, n)$ as the 'top right corner'.

Integer Sequences Let $s$ be an integer sequence of length $n$. We use the shorthand $' \min (s)$ ' for ' $\min _{i \in[n]} s(i)$ ' and ' $\max (s)$ ' for ' $\max _{i \in[n]} s(i)$ '; we use the following definitions. We let

$\operatorname{argmin}(s):=\{i \in[n] \mid s(i)=\min (s)\}$ and $\operatorname{argmax}(s):=\{i \in[n] \mid s(i)=\max (s)\}$

be the set of indices at whose positions there are the minimum and maximum element of $s$, respectively. Whenever we write $i \in \operatorname{argmin}(s)(j \in \operatorname{argmax}(s))$, then the choice of $i(j)$ can be arbitrary. In some places we require a canonical choice of the position of a minimum or maximum element, in which case we will always choose the smallest index. Formally, we let

$$
\operatorname{argmin} *(s):=\min \operatorname{argmin}(s), \text { and } \operatorname{argmax}(s):=\min \operatorname{argmax}(s) .
$$

The following definition contains two notions on pairs of integer sequences that are necessary for the definitions of domination and merges.

Definition 2.1 Let $r$ and $s$ be two integer sequences of the same length $n$.

(i) If for all $i \in[n], r(i) \leq s(i)$, then we write ' $r \leq s$ '.

(ii) We write $q=r+s$ for the integer sequence $q(1), \ldots, q(n)$ with $q(i)=r(i)+$ $s(i)$ for all $i \in[n]$.

Definition 2.2 (Extensions) Let $s$ be a sequence of length $n$. We define the set $E(s)$ of extensions of $s$ as the set of sequences that are obtained from $s$ by repeating each of its elements an arbitrary number of times, and at least once. Formally, we let

$$
E(s):=\left\{s_{1} \circ s_{2} \circ \cdots \circ s_{n} \mid \forall i \in[n]: l\left(s_{i}\right) \geq 1 \wedge \forall j \in\left[l\left(s_{i}\right)\right]: s_{i}(j)=s(i)\right\} .
$$

Definition 2.3 (Domination) Let $r$ and $s$ be integer sequences. We say that $r$ dominates $s$, in symbols ' $r \prec s$ ', if there are extensions $r^{*} \in E(r)$ and $s^{*} \in E(s)$ of the same length such that $r^{*} \leq s^{*}$. If $r \prec s$ and $s \prec r$, then we say that $r$ and $s$ are equivalent, and we write $r \equiv s$.

If $r$ is an integer sequence and $S$ is a set of integer sequences, then we say that $r$ dominates $S$, in symbols ' $r \prec S$ ', if for all $s \in S, r \prec s$.

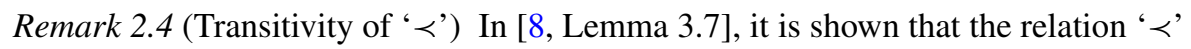
is transitive. As this is fairly intuitive, we may use this fact without stating it explicitly throughout this text.

A merge of two integer sequences $r$ and $s$ is the sum of an extension of $r$ and an extension of $s$ of the same length. 
Definition 2.5 (Merges) Let $r$ and $s$ be two integer sequences. We define the set of all merges of $r$ and $s$, denoted by $r \oplus s$, as $r \oplus s:=\left\{r^{*}+s^{*} \mid r^{*} \in E(r), s^{*} \in\right.$ $\left.E(s), l\left(r^{*}\right)=l\left(s^{*}\right)\right\}$.

\subsection{Typical Sequences}

We now define typical sequences, show how to construct them using linearly many integer operations, and restate several lemmas due to Bodlaender and Kloks [8] that will be used throughout this text.

Definition 2.6 Let $s=s(1), \ldots, s(n)$ be an integer sequence of length $n$. The typical sequence of $s$, denoted by $\tau(s)$, is obtained from $s$ by an exhaustive application of the following two operations:

Removal of Consecutive Repetitions If there is an index $i \in[n-1]$ such that $s(i)=$ $s(i+1)$, then we change the sequence $s$ from $s(1), \ldots, s(i), s(i+1), \ldots, s(n)$ to $s(1), \ldots, s(i), s(i+2), \ldots, s(n)$.

Typical Operation If there exist $i, j \in[n]$ such that $j-i \geq 2$ and for all $i \leq k \leq j, s(i) \leq s(k) \leq s(j)$, or for all $i \leq k \leq j, s(i) \geq s(k) \geq s(j)$, then we change the sequence $s$ from $s(1), \ldots, s(i), s(i+1), \ldots, s(j), \ldots, s(n)$ to $s(1), \ldots, s(i), s(j), \ldots, s(n)$, i.e., we remove all elements (strictly) between index $i$ and $j$.

To support intuition, we illustrate the rough shape of a typical sequence in Fig. 1. It is not difficult to see that the typical sequence can be computed using a quadratic amount of integer operations, by an exhaustive application of the definition. Here we discuss how to do it using a linear amount of integer operations. We may view a typical sequence $\tau(s)$ of an integer sequence $s$ as a subsequence of $s$. While $\tau(s)$ is unique, the choice of indices that induce $\tau(s)$ may not be unique. We show that we can find a set of indices that induce the typical sequence with help of the following structural proposition.

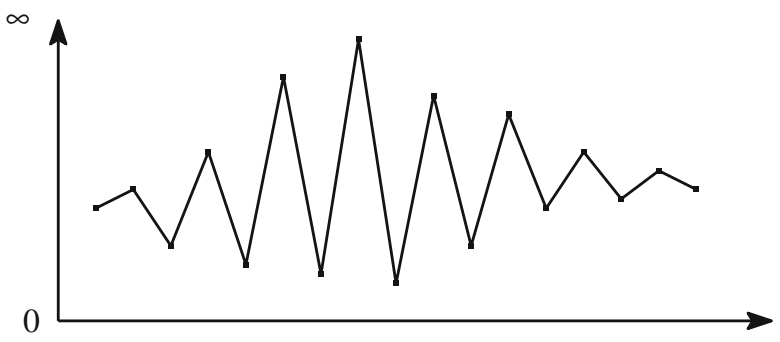

Fig. 1 Illustration of the shape of a typical sequence 
Proposition 2.7 Let $s$ be an integer sequence and let $i^{\star} \in\left\{\operatorname{argmin}^{*}(s)\right.$, $\left.\operatorname{argmax}^{*}(s)\right\}$. Let $1=: j_{0}<j_{1}<j_{2}<\ldots<j_{t}<j_{t+1}:=i^{\star}$ be pairwise distinct integers, such that $s\left(j_{0}\right), \ldots, s\left(j_{t+1}\right)$ are pairwise distinct. If for all $h \in[0 . . t]$,

- if $s\left(j_{h}\right)>s\left(j_{h+1}\right)$ then $j_{h}=\operatorname{argmax}^{*}\left(s\left[1 . . j_{h+1}\right]\right)$ and $j_{h+1}=$ $\operatorname{argmin} *\left(s\left[1 . . j_{h+1}\right]\right)$, and

- if $s\left(j_{h}\right)<s\left(j_{h+1}\right)$ then $j_{h}=\operatorname{argmin}^{*}\left(s\left[1 . . j_{h+1}\right]\right)$ and $j_{h+1}=$ $\operatorname{argmax}^{*}\left(s\left[1 . . j_{h+1}\right]\right)$,

then the typical sequence of $s$ restricted to $\left[i^{\star}\right]$ is equal to $s\left(j_{0}\right), s\left(j_{1}\right), \ldots$, $s\left(j_{t}\right), s\left(j_{t+1}\right)$.

Proof First, we observe that by the choice made in the definition of argmin* and $\operatorname{argmax}$,

for each $h \in[0 . .(t+1)]$ there is no $i<j_{h}$ such that $s(i)=s\left(j_{h}\right)$.

We prove the following statement. Under the stated conditions, for a given $h \in$ $[0 . . t+1]$, the typical sequence of $s$ restricted to $\left[j_{h} . . i^{\star}\right]$ is equal to $s\left(j_{h}\right), s\left(j_{h+1}\right), \ldots$, $s\left(j_{t+1}\right)$. The proposition then follows from the case $h=0$. The proof is by induction on $d:=(t+1)-h$. For $d=0$, it trivially holds since the minimum and the maximum element are always part of the typical sequence, and since $\left[j_{t+1} . . i^{\star}\right]=\left\{i^{\star}\right\}$.

Now suppose $d>0$, and for the induction hypothesis that the claim holds for $d-1$. Suppose that $s\left(j_{h}\right)>s\left(j_{h+1}\right)$, meaning that $j_{h}=\operatorname{argmax} *\left(s\left[1 . . j_{h+1}\right]\right)$, and $j_{h+1}=\operatorname{argmin}^{*}\left(s\left[1 . . j_{h+1}\right]\right)$, the other case is symmetric. By the induction hypothesis, the typical sequence of $s$ restricted to $\left[j_{h+1} . . i^{\star}\right]$ is equal to $s\left(j_{h+1}\right), \ldots, s\left(j_{t+1}\right)$, in particular it implies that $s\left(j_{h+1}\right)$ is an element of the typical sequence. To prove the induction step, we have to show that the typical sequence of $s$ restricted to [ $\left.j_{h} . . j_{h+1}\right]$ is equal to $s\left(j_{h}\right), s\left(j_{h+1}\right)$. We first argue that if there is an element of the typical sequence in $\left[j_{h} . .\left(j_{h+1}-1\right)\right]$, then it must be equal to $s\left(j_{h}\right)$. By $(2)$, we have that there is no $i<j_{h+1}$ such that $s(i)=s\left(j_{h+1}\right)$, and together with the fact that $s\left(j_{h+1}\right)$ is the minimum value of $s\left[1 . . j_{h+1}\right]$, we conclude that $\left[j_{h} . .\left(j_{h+1}-1\right)\right]$ cannot contain any element of the typical sequence that is equal to $s\left(j_{h+1}\right)$. Next, since the typical operation removes all elements $i \in\left[\left(j_{h}+1\right) . .\left(j_{h+1}-1\right)\right]$ with $s\left(j_{h}\right)>s(i)>s\left(j_{h+1}\right)$, and since $j_{h}=\operatorname{argmax}^{*}\left(s\left[1 . . j_{h+1}\right]\right)$, the only elements from $\left[j_{h} . .\left(j_{h+1}-1\right)\right]$ that the typical sequence may contain have value $s\left(j_{h}\right)$.

It remains to argue that $s\left(j_{h}\right)$ is indeed an element of the typical sequence. Suppose not, then there are indices $i, i^{\prime}$ with $i<j_{h}<i^{\prime}$, such that either $s(i) \leq s\left(j_{h}\right) \leq s\left(i^{\prime}\right)$, or $s(i) \geq s\left(j_{h}\right) \geq s\left(i^{\prime}\right)$, and we may assume that at least one of the inequalities is strict in each case. For the latter case, since $j_{h}=\operatorname{argmax}^{*}\left(s\left[1 . . j_{h+1}\right]\right)$, we would have that $s(i)=s\left(j_{h}\right)$, which is a contradiction to (2). Hence, we may assume that $s(i) \leq s\left(j_{h}\right) \leq s\left(i^{\prime}\right)$. There are two cases to consider: $i^{\prime} \in\left[\left(j_{h}+1\right) . . j_{h+1}\right]$, and $i^{\prime}>$ $j_{h+1}$. If $i^{\prime} \in\left[\left(j_{h}+1\right) . . j_{h+1}\right]$, then $s\left(i^{\prime}\right)=s\left(j_{h}\right)$, as $s\left(j_{h}\right)=\operatorname{argmax}\left(s\left[1 . . j_{h+1}\right]\right)$. We can conclude that in this case, the typical sequence must contain an element equal to $s\left(i^{\prime}\right)$, and hence equal to $s\left(j_{h}\right)$. If $i^{\prime}>j_{h+1}$, then the typical operation corresponding to $i$ and $i^{\prime}$ also removes $s\left(j_{h+1}\right)$, a contradiction with the induction hypothesis which 
asserts that $s\left(j_{h+1}\right)$ is part of the typical sequence induced by $\left[j_{h+1} . . i^{\star}\right]$. We can conclude that $s\left(j_{h}\right)$ is part of the typical sequence, finishing the proof.

From the previous proposition, we have the following consequence about the structure of typical sequences ending in the minimum element, which will be useful in the proof of Lemma 3.10.

Corollary 2.8 Let $t$ be a typical sequence of length $n$ such that $n \in \operatorname{argmin}(t)$. Then, for each $k \in\left[\left\lfloor\frac{n}{2}\right\rfloor\right], n-2 k+1 \in \operatorname{argmax}(t[1 . .(n-2 k+1)])$ and $n-2 k \in$ $\operatorname{argmin}(t[1 . .(n-2 k)])$.

Equipped with Proposition 2.7, we can now proceed and give the algorithm that computes a typical sequence of an integer sequence using linearly many integer operations.

Lemma 2.9 Let $s$ be an integer sequence of length $n$. Then, one can compute $\tau(s)$, the typical sequence of $s$, in $\mathcal{O}(n)$ integer operations.

Proof First, we check for each $i \in[n-1]$ whether $s(i)=s(i+1)$, and if we find such an index $i$, we remove $s(i)$. We assume from now on that after these modifications, $s$ has at least two elements, otherwise it is trivial. As observed above, the typical sequence of $s$ contains $\min (s)$ and $\max (s)$. A closer look reveals the following observation.

Observation 2.9.1 Let $i^{\star}:=\min \operatorname{argmin}(s) \cup \operatorname{argmax}(s)$ and $k^{\star}:=\max \operatorname{argmin}(s) \cup$ $\operatorname{argmax}(s)$.

(i) If $i^{\star} \in \operatorname{argmin}(s)$ and $k^{\star} \in \operatorname{argmax}(s)$ or $i^{\star} \in \operatorname{argmax}(s)$ and $k^{\star} \in \operatorname{argmin}(s)$, then $\tau(s)$ restricted to $\left[i^{\star} . . k^{\star}\right]$ is equal to $s\left(i^{\star}\right), s\left(k^{\star}\right)$.

(ii) If $\left\{i^{\star}, k^{\star}\right\} \subseteq \operatorname{argmin}(s)$, then $\tau(s)$ restricted to $\left[i^{\star} . . k^{\star}\right]$ is equal to $s\left(i^{\star}\right), \max (s), s\left(k^{\star}\right)$.

(iii) If $\left\{i^{\star}, k^{\star}\right\} \subseteq \operatorname{argmax}(s)$, then $\tau(s)$ restricted to $\left[i^{\star} . . k^{\star}\right]$ is equal to $s\left(i^{\star}\right), \min (s), s\left(k^{\star}\right)$.

Let $i^{\star}:=\min \operatorname{argmin}(s) \cup \operatorname{argmax}(s)$ and $k^{\star}:=\max \operatorname{argmin}(s) \cup \operatorname{argmax}(s)$. Using Observation 2.9.1, it remains to determine the indices that induce the typical sequence on $s\left[1 . . i^{\star}\right]$ and on $s\left[k^{\star} . . n\right]$. To find the indices that induce the typical sequence on $s\left[1 . . i^{\star}\right]$, we will describe a marking procedure that marks a set of indices satisfying the preconditions of Proposition 2.7. Next, we observe that $n-k^{\star}$ is the smallest index of any occurrence of $\min (s)$ or $\max (s)$ in the reverse sequence of $s$, therefore a symmetric procedure, again using Proposition 2.7, yields the indices that induce $\tau(s)$ on $s\left[k^{\star} . . n\right]$. 


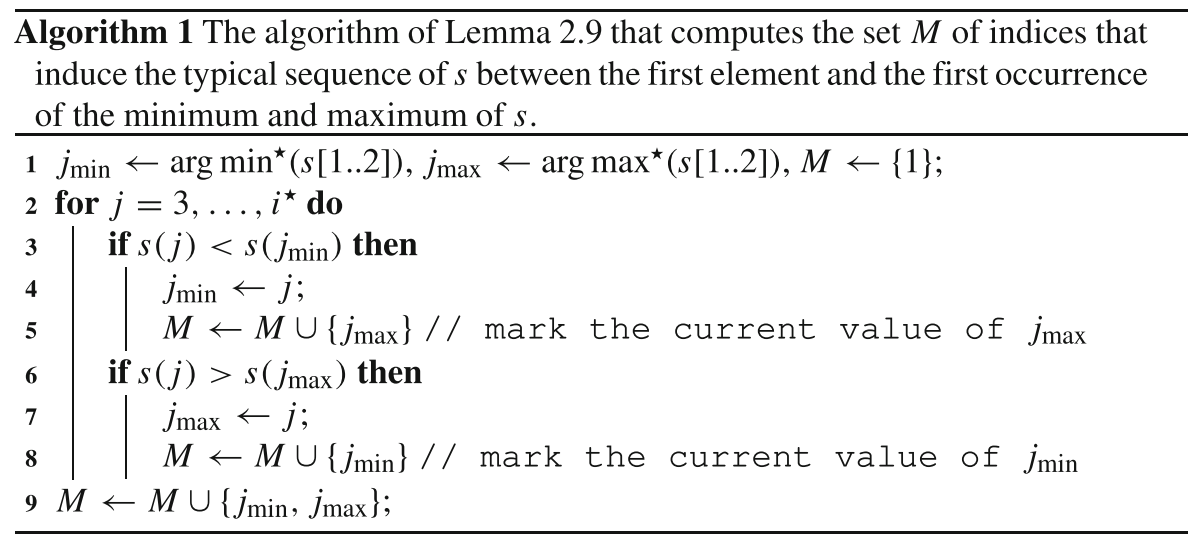

We execute Algorithm 1, which processes the integer sequence $s\left[1 . . i^{\star}\right]$ from the first to the last element, storing two counters $j_{\min }$ and $j_{\max }$ that store the leftmost position of the smallest and of the greatest element seen so far, respectively. Whenever a new minimum is encountered, we mark the current value of the index $j_{\max }$, as this implies that $s\left(j_{\max }\right)$ has to be an element of the typical sequence. Similarly, when encountering a new maximum, we mark $j_{\min }$. These marked indices are stored in a set $M$, which at the end of the algorithm contains the indices that induce $\tau(s)$ on $\left[1 . . i^{\star}\right]$. This, i.e., the correctness of the procedure, will now be argued via Proposition 2.7.

Claim 2.9.2 The set $M$ of indices marked by the above procedure induce $\tau(s)$ on $\left[1 . . i^{\star}\right]$.

Proof Let $M=\left\{j_{0}, j_{1}, \ldots, j_{t+1}\right\}$ be such that for all $h \in[0 . . t], j_{h}<j_{h+1}$. We prove that $j_{0}, \ldots, j_{t+1}$ meet the preconditions of Proposition 2.7. First, we observe that the above algorithm marks both the index 1 and index $i^{\star}$, in particular that $j_{0}=1$ and $j_{t+1}=i^{\star}$.

We verify that the indices $j_{0}, \ldots, j_{t+1}$ satisfy the property that for each $h \in$ $[0 . .(t+1)]$, the index $j_{h}$ is the leftmost (i.e., smallest) index whose value is equal to $s\left(j_{h}\right)$ : whenever an index is added to the marked set, it is because in some iteration, the element at its position was either strictly greater than the greatest previously seen element, or strictly smaller than the smallest previously seen element. (This also ensures that $s\left(j_{0}\right), \ldots, s\left(j_{t+1}\right)$ are pairwise distinct.)

We additionally observe that if we have two indices $\ell_{1}$ and $\ell_{2}$ such that $\ell_{2}$ is the index that the algorithm marked right after it marked $\ell_{1}$, then either $\ell_{1}$ was $j_{\min }$ and $\ell_{2}$ was $j_{\max }$ or vice versa: when updating $j_{\min }$, we mark $j_{\max }$, and when updating $j_{\max }$, we mark $j_{\min }$. This lets us conclude that when we have two indices $j_{h}, j_{h+1}$ such that $s\left(j_{h}\right)<s\left(j_{h+1}\right)$, then $j_{h}$ was equal to $j_{\min }$ when it was marked, and $j_{h+1}$ was $j_{\max }$ when it was marked.

We are ready to prove that $j_{0}, \ldots, j_{t+1}$ satisfy the precondition of Proposition 2.7. Suppose for a contradiction that for some $h \in[0 . . t+1], j_{h}$ violates this property. Assume that $s\left(j_{h}\right)<s\left(j_{h+1}\right)$ and note that the other case is symmetric. The previous 
paragraph lets us conclude that $j_{h}$ was equal to $j_{\min }$ when it was marked, and that $j_{h+1}$ was $j_{\max }$ when it was marked.

We either have that $j_{h} \neq \operatorname{argmin} *\left(s\left[1 . . j_{h+1}\right]\right)$ or that $j_{h+1} \neq \operatorname{argmax}^{*}\left(s\left[1 . . j_{h+1}\right]\right)$. Suppose the latter holds. This immediately implies that there is some $j^{*} \in\left[j_{h+1}-1\right]$ such that $s\left(j^{*}\right)>s\left(j_{h+1}\right)$, which implies that $j_{\max }$ would never have been set to $j_{h+1}$ and hence $j_{h+1}$ would have never been marked. Suppose the former holds, i.e., $j_{h} \neq \operatorname{argmin}^{*}\left(s\left[1 . . j_{h+1}\right]\right)$, for an illustration of the following argument see Fig. 2. Let $j^{*}:=\operatorname{argmin}^{*}\left(s\left[1 . . j_{h+1}\right]\right)$. If $j^{*}<j_{h}$, then at iteration $j_{h}, s\left(j_{\min }\right) \leq s\left(j_{h}\right)$, so $j_{\min }$ would never have been set to $j_{h}$, and hence, $j_{h}$ would never have been marked. We may assume that $j^{*}>j_{h}$. Since $j_{h}$ was marked, there is some $\ell>j_{h}$ that triggered $j_{h}$ being marked. This also means that at that iteration $s(\ell)$ was greater than the previously observed maximum, so we may assume that $s(\ell)>s\left(j_{h}\right)$. We also may assume that $\ell \leq j_{h+1}$. If $j^{*} \in\left[\left(j_{h}+1\right)\right.$..( $\left.(\ell-1)\right]$, then the algorithm would have updated $j_{\min }$ to $j^{*}$ in that iteration, before marking $j_{h}$, and for the case $j^{*} \in\left[(\ell+1) . .\left(j_{h+1}-1\right)\right]$ we observe that $\ell \neq j_{h+1}$, and that the algorithm would mark $\ell$ as the next index instead of $j_{h+1}$.

This establishes the correctness of the algorithm. We observe that each iteration takes $\mathcal{O}(1)$ comparisons of numbers in $s$, and that there are $\mathcal{O}(n)$ iterations.

We summarize several lemmas from [8] regarding integer sequences and typical sequences that we will use in this work.

Lemma 2.10 (Bodlaender and Kloks [8]) Let $r$ and $s$ be two integer sequences.

(i) (Cor. 3.11 in [8]). We have that $r \prec s$ if and only if $\tau(r) \prec \tau(s)$.

(ii) (Lem. 3.13 in [8]). Suppose $r$ and $s$ are of the same length and let $y=r+s$. Let $r_{0} \prec r$ and $s_{0} \prec s$. Then there is an integer sequence $y_{0} \in r_{0} \oplus s_{0}$ such that $y_{0} \prec y$.

(iii) (Lem. 3.14 in [8]). Let $q \in r \oplus s$. Then, there is an integer sequence $q^{\prime} \in$ $\tau(r) \oplus \tau(s)$ such that $q^{\prime} \prec q$.

(iv) (Lem. 3.15 in [8]). Let $q \in r \oplus s$. Then, there is an integer sequence $q^{\prime} \in r \oplus s$ with $\tau\left(q^{\prime}\right)=\tau(q)$ and $l\left(q^{\prime}\right) \leq l(r)+l(s)-1$.

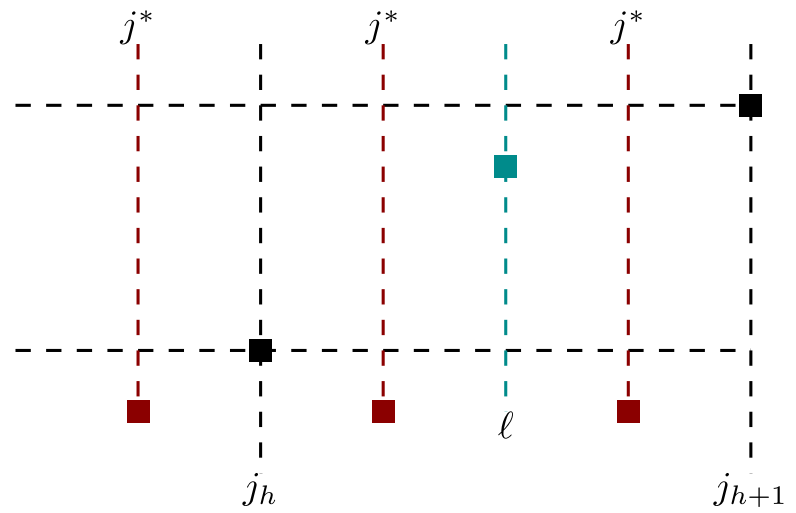

Fig. 2 Illustration of the final argument in the proof of Claim 2.9.2. We assume that $s\left(j_{h}\right)<s\left(j_{h+1}\right)$, and mark the possible positions for $j^{*}=\operatorname{argmin} *\left(s\left[1 . . j_{h+1}\right]\right)$ with $j^{*} \neq j_{h}$ 
(v) (Lem. 3.19 in [8]). Let $r^{\prime}$ and $s^{\prime}$ be two more integer sequences. If $r^{\prime} \prec r$ and $s^{\prime} \prec s$, then $r^{\prime} \circ s^{\prime} \prec r \circ s$.

\subsection{Directed Acyclic Graphs}

A directed graph (or digraph) $G$ is a pair of a set of vertices $V(G)$ and a set of ordered pairs of vertices, called $\operatorname{arcs}, A(G) \subseteq V(G) \times V(G)$. (If $A(G)$ is a multiset, we call $G$ multidigraph.) We say that an $\operatorname{arc} a=(u, v) \in A(G)$ is directed from $u$ to $v$, and we call $u$ the tail of $a$ and $v$ the head of $a$. A sequence of vertices $v_{1}, \ldots, v_{r}$ is called a walk in $G$ if for all $i \in[r-1],\left(v_{i}, v_{i+1}\right) \in A(G)$. A cycle is a walk $v_{1}, \ldots, v_{r}$ with $v_{1}=v_{r}$ and all vertices $v_{1}, \ldots, v_{r-1}$ pairwise distinct. If $G$ does not contain any cycles, then we call $G$ acyclic or a directed acyclic graph, DAG for short.

Let $G$ be a DAG on $n$ vertices. A topological order of $G$ is a linear order $\pi: V(G) \rightarrow[n]$ such that for all $\operatorname{arcs}(u, v) \in A(G), \pi(u)<\pi(v)$. We denote the set of all topological orders of $G$ by $\Pi(G)$. We now define the width measures studied in this work. Note that we restrict the orders of the vertices that we consider to topological orders.

Definition 2.11 Let $G$ be a directed acyclic graph and let $\pi \in \Pi(G)$ be a topological order of $G$.

(i) The cutwidth of $\pi$ is cutw $(\pi):=\max _{i \in[n-1]} \mid\{(u, v) \in A(G) \mid \pi(u) \leq i \wedge \pi(v)>i\}$.

(ii) The modified cutwidth of $\pi$ is $\operatorname{mcutw}(\pi):=\max _{i \in[n]} \mid\{(u, v) \in A(G) \mid$ $\pi(u)<i \wedge \pi(v)>i\}$.

We define the cutwidth and modified cutwidth of a directed acyclic graph $G$ as the minimum of the respective measure over all topological orders of $G$.

We now introduce series parallel digraphs. Note that the following definition coincides with the notion of 'edge series-parallel multidigraphs' in [25]. For an illustration see Fig. 3.

Definition 2.12 (Series Parallel Digraph (SPD)) A (multi-)digraph $G$ with an ordered pair of distinct terminals $(s, t) \in V(G) \times V(G)$ is called series parallel digraph $(S P D)$, often denoted by $(G,(s, t))$, if one of the following hold.
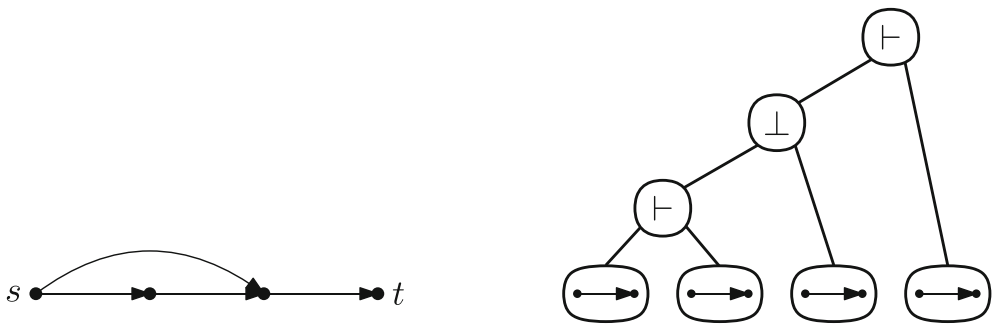

Fig. 3 A series parallel digraph $G$ on the left, and a decomposition tree that yields $G$ on the right 
(i) $(G,(s, t))$ is a single arc directed from $s$ to $t$, i.e., $V(G)=\{s, t\}, A(G)=$ $\{(s, t)\}$.

(ii) $(G,(s, t))$ can be obtained from two series parallel digraphs $\left(G_{1},\left(s_{1}, t_{1}\right)\right)$ and $\left(G_{2},\left(s_{2}, t_{2}\right)\right)$ by one of the following operations.

(a) Series Composition. $(G,(s, t))$ is obtained by taking the disjoint union of $G_{1}$ and $G_{2}$, identifying $t_{1}$ and $s_{2}$, and letting $s=s_{1}$ and $t=t_{2}$. In this case we write $(G,(s, t))=\left(G_{1},\left(s_{1}, t_{1}\right)\right) \bullet\left(G_{2},\left(s_{2}, t_{2}\right)\right)$ or simply $G=G_{1} \bullet G_{2}$.

(b) Parallel Composition. $(G,(s, t))$ is obtained by taking the disjoint union of $G_{1}$ and $G_{2}$, identifying $s_{1}$ and $s_{2}$, and identifying $t_{1}$ and $t_{2}$, and letting $s=s_{1}=s_{2}$ and $t=t_{1}=t_{2}$. In this case we write $(G,(s, t))=$ $\left(G_{1},\left(s_{1}, t_{1}\right)\right) / /\left(G_{2},\left(s_{2}, t_{2}\right)\right)$, or simply $G=G_{1} / / G_{2}$.

It is not difficult to see that each series parallel digraph is acyclic. One can naturally associate a notion of decomposition trees with series parallel digraphs as follows. A decomposition tree $T$ is a rooted and ordered binary tree whose leaves are labeled with a single arc, and each internal node $t \in V(T)$ with left child $\ell$ and right child $r$ is either a series node or a parallel node. We then associate an SPD $G_{t}$ with each node $t \in V(T)$. If $t$ is a leaf, then $G_{t}$ is a single arc oriented from one terminal to the other. If $t$ is an internal node, then $G_{t}$ is $G_{\ell} \bullet G_{r}$ if $t$ is a series node and $G_{\ell} / / G_{r}$ if $t$ is a parallel node. It is clear that for each SPD $G$, there is a decomposition tree $T$ with root $\mathfrak{r}$ such that $G=G_{\mathfrak{r}}$. In that case we say that $T$ yields $G$. Valdes et al. [25] have shown that one can decide in linear time whether a directed graph $G$ is an SPD and if so, find a decomposition tree that yields $G$.

Theorem 2.13 (Valdes et al. [25]) Let $G$ be a directed graph on $n$ vertices and $m$ arcs. There is an algorithm that decides in time $\mathcal{O}(n+m)$ whether $G$ is a series parallel digraph and if so, it outputs a decomposition tree that yields $G$.

\section{The Merge Dominator Lemma}

In this section we prove the main technical result of this work. It states that given two integer sequences, one can find a merge that dominates all merges of those two sequences using linearly many integer operations.

Lemma 3.1 (Merge Dominator Lemma) Let $r$ and $c$ be integer sequence of length $m$ and $n$, respectively. There exists a dominating merge of $r$ and $c$, i.e., an integer sequence $t \in r \oplus c$ such that $t \prec r \oplus c$, and this dominating merge can be computed using $\mathcal{O}(m+n)$ integer operations.

Outline of the Proof of the Merge Dominator Lemma First, we show that we can restrict our search to finding a dominating path in a matrix that, roughly speaking, contains all merges of $r$ and $c$ of length at most $l(r)+l(c)-1$. The goal of this step is mainly to increase the intuitive insight to the proofs in this section. Next, we prove the 'Split Lemma' (Lemma 3.7 in Section 3.2) which asserts that we can obtain a 
dominating path in our matrix $M$ by splitting $M$ into a submatrix $M_{1}$ that lies in the 'bottom left' of $M$ and another submatrix $M_{2}$ in the 'top right' of $M$ along a minimum row and a minimum column, and appending a dominating path in $M_{2}$ to a dominating path in $M_{1}$. In $M_{1}$, the last row and column are a minimum row and column, respectively, and in $M_{2}$, the first row and column are a minimum row and column, respectively. This additional structure will be exploited in Section 3.3 where we prove the 'Chop Lemmas' that come in two versions. The 'bottom version' (Lemma 3.10) shows that in $M_{1}$, we can find a dominating path by repeatedly chopping away the last two rows or columns and remembering a vertical or horizontal length- 2 path. The 'top version' (Corollary 3.12) is the symmetric counterpart for $M_{2}$. The proofs of the Chop Lemmas only hold when $r$ and $c$ are typical sequences, and in Section 3.4 we present the 'Split-and-Chop Algorithm' that computes a dominating path in a merge matrix of two typical sequences. Finally, in Section 3.5, we generalize this result to arbitrary integer sequences, using the Split-and-Chop Algorithm and one additional construction.

\subsection{The Merge Matrix, Paths, and Non-Diagonality}

Let us begin by defining the basic notions of a merge matrix and paths in matrices.

Definition 3.2 (Merge Matrix) Let $r$ and $c$ be two integer sequences of length $m$ and $n$, respectively. Then, the merge matrix of $r$ and $c$ is an $m \times n$ integer matrix $M$ such that for $(i, j) \in[m] \times[n], M[i, j]=r(i)+c(j)$.

We would like to point out that the following definition of a path in a matrix can be viewed as a special case of the notion of lattice paths, see [22], or [14] for a related application.

Definition 3.3 (Path in a Matrix) Let $M$ be an $m \times n$ matrix. A path in $M$ is a sequence $p(1), \ldots, p(\ell)$ of indices from $M$ such that

(i) $p(1)=(1,1)$ and $p(\ell)=(m, n)$, and

(ii) for $h \in[\ell-1]$, let $p(h)=(i, j)$; then, $p(h+1) \in\{(i+1, j),(i, j+1),(i+$ $1, j+1)\}$.

We denote by $\mathcal{P}(M)$ the set of all paths in $M$. A sequence $p(1), \ldots, p(\ell)$ that satisfies the second condition but not necessarily the first is called a partial path in $M$. For two paths $p, q \in \mathcal{P}(M)$, we may simply say that $p$ dominates $q$, if $M[p]$ dominates $M[q] .{ }^{4}$ We also write $p \prec \mathcal{P}(M)$ to express that for each path $q \in \mathcal{P}(M), p \prec q$.

A (partial) path is called non-diagonal if the second condition is replaced by the following.

(ii)' For $h \in[\ell-1]$, let $p(h)=(i, j)$; then, $p(h+1) \in\{(i+1, j),(i, j+1)\}$.

\footnotetext{
${ }^{4}$ Recall that by (1) on page 4 , for a (partial) path $p$ in a matrix $M, M[p]=$ $M[p(1)], M[p(2)], \ldots, M[p(l(p))]$.
} 
An extension $e$ of a path $p$ in a matrix $M$ is as well a sequence of indices of $M$, and we again denote the corresponding integer sequence by $M[e]$. A consequence of Lemma 2.10(i) and (iv) is that we can restrict ourselves to all paths in a merge matrix when trying to find a dominating merge of two integer sequences: it is clear from the definitions that in a merge matrix $M$ of integer sequences $r$ and $c, \mathcal{P}(M)$ contains all merges of $r$ and $c$ of length at most $l(r)+l(c)-1$. Furthermore, suppose that there is a merge $q \in r \oplus s$ such that $q \prec r \oplus s$ and $l(q)>l(r)+l(s)-1$. By Lemma 2.10(iv), there is a merge $q^{\prime} \in r \oplus s$ such that $l\left(q^{\prime}\right) \leq l(r)+l(s)-1$, and $\tau\left(q^{\prime}\right)=\tau(q)$. The latter yields $\tau\left(q^{\prime}\right) \equiv \tau(q)$ and therefore, by Lemma $2.10(\mathrm{i}), q^{\prime} \equiv q$, in particular, $q^{\prime} \prec q \prec r \oplus s$.

Corollary 3.4 Let $r$ and $c$ be integer sequences and $M$ be the merge matrix of $r$ and $c$. There is a dominating merge in $r \oplus c$, i.e., an integer sequence $t \in r \oplus c$ such that $t \prec r \oplus c$, if and only if there is a dominating path in $M$, i.e., a path $p \in \mathcal{P}(M)$ such that $p \prec \mathcal{P}(M)$.

We now consider a type of merge that corresponds to non-diagonal paths in the merge matrix. These merges will be used in a construction presented in Section 3.5, and in the algorithmic applications of the Merge Dominator Lemma given in Section 4. For two integer sequences $r$ and $s$, we denote by $r \boxplus s$ the set of all non-diagonal merges of $r$ and $s$, which are not allowed to have 'diagonal' steps: we have that for all $t \in r \boxplus s$ and all $i \in[l(t)-1]$, if $t(i)=r\left(i_{r}\right)+s\left(i_{s}\right)$, then $t(i+1) \in\left\{r\left(i_{r}+1\right)+s\left(i_{s}\right), r\left(i_{r}\right)+s\left(i_{s}+1\right)\right\}$. As each non-diagonal merge directly corresponds to a non-diagonal path in the merge matrix (and vice versa), we can consider a non-diagonal path in a merge matrix to be a non-diagonal merge and vice versa. We now show that for each merge that uses diagonal steps, there is always a non-diagonal merge that dominates it.

Lemma 3.5 Let $r$ and $s$ be two integer sequences of length $m$ and $n$, respectively. For any merge $q \in r \oplus s$, there is a non-diagonal merge $q^{\prime} \in r \boxplus s$ such that $q^{\prime} \prec q$. Furthermore, given $q, q^{\prime}$ can be found using $\mathcal{O}(m+n)$ integer operations.

Proof This can be shown by the following local observation. Let $i \in[l(q)-1]$ be such that $q(i), q(i+1)$ is a diagonal step, i.e., there are indices $i_{r} \in[l(r)-1]$ and $i_{s} \in[l(s)-1]$ such that $q(i)=r\left(i_{r}\right)+s\left(i_{s}\right)$ and $q(i+1)=r\left(i_{r}+1\right)+s\left(i_{s}+1\right)$. Then, we insert the element $x:=\min \left\{r\left(i_{r}\right)+s\left(i_{s}+1\right), r\left(i_{r}+1\right)+s\left(i_{s}\right)\right\}$ between $q(i)$ and $q(i+1)$. Since

$$
x \leq \max \left\{r\left(i_{r}\right)+s\left(i_{s}\right), r\left(i_{r}+1\right)+s\left(i_{s}+1\right)\right\}=: y,
$$

we can repeat $y$ twice in an extension of $q$ so that one of the occurrences aligns with $x$, and we have that in this position, the value of $q^{\prime}$ is at most the value of the extension of $q$.

Let $q^{\prime}$ be the sequence obtained from $q$ by applying this operation to all diagonal steps, then by the observation just made, we have that $q^{\prime} \prec q$. It is clear that this can be implemented using $\mathcal{O}(m+n)$ integer operations. 
Next, we define two special paths in a matrix $M$ that will reappear in several places throughout this section. These paths can be viewed as the 'corner paths', where the first one follows the first row until it hits the last column and then follows the last column $\left(p_{\lrcorner}(M)\right)$, and the second one follows the first column until it hits the last row and then follows the last row $(p\ulcorner(M))$. Formally, we define them as follows:

$$
\begin{aligned}
& p_{\lrcorner}(M):=(1,1),(1,2), \ldots,(1, n),(2, n), \ldots,(m, n) \\
& p\ulcorner(M):=(1,1),(2,1) \ldots,(m, 1),(m, 2), \ldots,(m, n)
\end{aligned}
$$

We use the shorthands ' $p\lrcorner$ ' for ' $p\lrcorner(M)$ ' and ' $p\ulcorner$ ' for ' $p\ulcorner(M)$ ' whenever $M$ is clear from the context.

For instance, these paths appear in the following special cases of the Merge Dominator Lemma, which will be useful for several proofs in this section.

Lemma 3.6 Let $r$ and $c$ be integer sequences of length $m$ and $n$, respectively, and let $M$ be the merge matrix of $r$ and $c$. Let $i \in \operatorname{argmin}(r)$ and $j \in \operatorname{argmin}(c)$.

(i) If $i=1$ and $j=n$, then $p_{\lrcorner}$dominates all paths in $M$, i.e., $p_{\lrcorner} \prec \mathcal{P}(M)$.

(ii) If $i=m$ and $j=1$, then $p\ulcorner$ dominates all paths in $M$, i.e., $p\ulcorner\prec \mathcal{P}(M)$.

Proof (i) For an illustration of this proof see Fig. 4. Let $q$ be any path in $M$ and let $t^{*}:=\operatorname{argmax}^{*}(q)$. Let furthermore $q\left(t^{*}\right)=\left(t_{r}^{*}, t_{c}^{*}\right)$. We divide $\left.p\right\lrcorner$ and $q$ in three consecutive parts each to show that $p\lrcorner$ dominates $q$.

- We let $p_{\lrcorner}^{1}:=p_{\lrcorner}(1), \ldots, p_{\lrcorner}\left(t_{c}^{*}-1\right)$ and $q_{1}:=q(1), \ldots, q\left(t^{*}-1\right)$.

- We let $p_{\lrcorner}^{2}:=p_{\lrcorner}\left(t_{c}^{*}\right), \ldots, p_{\lrcorner}\left(n+t_{r}^{*}-1\right)$ and $q_{2}:=q\left(t^{*}\right)$.

- We let $p_{\lrcorner}:=p_{\lrcorner}\left(n+t_{r}^{*}\right), \ldots, p_{\lrcorner}(m+n-1)$ and $q_{3}:=q\left(t^{*}+1\right), \ldots, q(l(q))$.

Fig. 4 Situation in the proof of Lemma 3.6(i). The dot (light/dark) within each element of the corner path $p_{\lrcorner}$indicates with which elements of the path $q$ it is 'matched up' in the extensions constructed in the proof

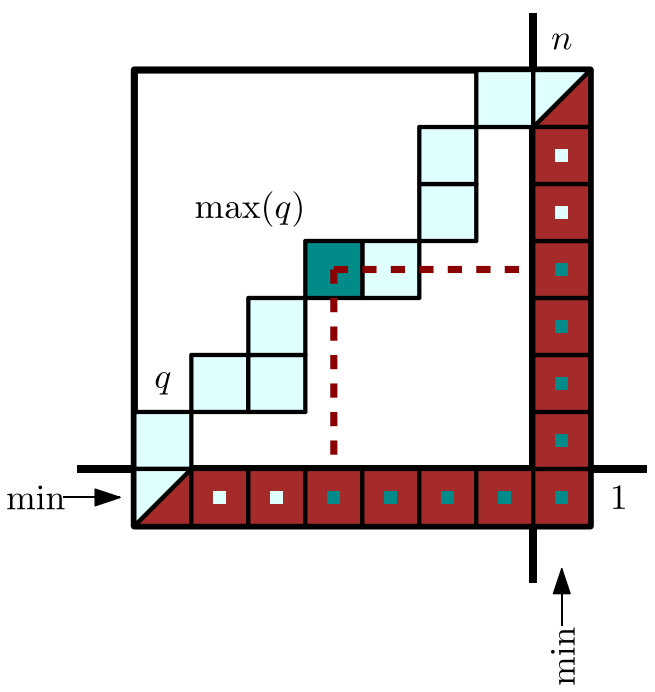


Since $r(1)$ is a minimum row in $M$, we have that for all $(k, \ell) \in[m] \times[n]$, $M[1, \ell] \leq M[k, \ell]$. This implies that there is an extension $e_{1}$ of $p_{\lrcorner}^{1}$ of length $t^{*}-1$ such that $M\left[e_{1}\right] \leq M\left[q_{1}\right]$. To clarify, the extension $e_{1}$ can be obtained as follows. For each $h \in\left[t^{*}-1\right]$, let $q(h)=\left(h_{r}, h_{c}\right)$. Then, at position $h, e_{1}$ contains $\left(1, h_{c}\right)$. Since $q$ is a path, $e_{1}$ is indeed an extension of $p_{\lrcorner}^{1}$. Similarly, there is an extension $e_{3}$ of $p_{\lrcorner}^{3}$ of length $l(q)-t^{*}$ such that $M\left[e_{3}\right] \leq M\left[q_{3}\right]$. Finally, let $f_{2}$ be an extension of $q_{2}$ that repeats its only element, $q\left(t^{*}\right), n-t_{c}^{*}+t_{r}^{*}$ times. Since $M\left[q\left(t^{*}\right)\right]$ is the maximum element on the sequence $M[q]$ and $r(1)$ is a minimum row and $c(n)$ a minimum column in $M$, we have that $M\left[p_{\lrcorner}^{2}\right] \leq M\left[f_{2}\right]$ : for all $h \in\left[t_{c}^{*} . . n\right]$, there is some $h_{q} \in[l(q)]$ such that $q\left(h_{q}\right)=(j, h)$ for some row $j$, so $M\left[p_{\lrcorner}(h)\right]=$ $M[1, h] \leq M[j, h]=M\left[q\left(h_{q}\right)\right] \leq M\left[q\left(t^{*}\right)\right]$ (similarly for all $\left.h \in\left[n . .\left(n+t_{r}^{*}-1\right)\right]\right)$.

We define an extension $e$ of $p_{\lrcorner}$as $e:=e_{1} \circ p_{\lrcorner}^{2} \circ e_{3}$ and an extension $f$ of $q$ as $f:=q_{1} \circ f_{2} \circ q_{3}$. Note that $l(e)=l(f)=l(q)+n+t_{r}^{*}-\left(t_{c}^{*}+1\right)$, and by the above discussion, we have that $M[e] \leq M[f]$. (ii) follows from a symmetric argument.

\subsection{The Split Lemma}

In this section we prove the first main step towards the Merge Dominator Lemma. It is fairly intuitive that a dominating merge has to contain the minimum element of a merge matrix. (Otherwise, there is a path that cannot be dominated by that merge.) The Split Lemma states that in fact, we can split the matrix $M$ into two smaller submatrices, one that has the minimum element in the top right corner, and one that has the minimum element in the bottom left corner, compute a dominating path for each of them, and paste them together to obtain a dominating path for $M$.

Lemma 3.7 (Split Lemma) Let $r$ and $c$ be integer sequences of length $m$ and $n$, respectively, and let $M$ be the merge matrix of $r$ and $c$. Let $i \in \operatorname{argmin}(r)$ and $j \in$ $\operatorname{argmin}(c)$. Let $M_{1}:=M[1 . . i, 1 . . j]$ and $M_{2}:=M[i . . m, j . . n]$ and for all $h \in$ [2], let $p_{h} \in \mathcal{P}\left(M_{h}\right)$ be a dominating path in $M_{h}$, i.e., $p_{h} \prec \mathcal{P}\left(M_{h}\right)$. Then, $p_{1} \circ p_{2}$ is a dominating path in $M$, i.e., $p_{1} \circ p_{2} \prec \mathcal{P}(M)$.

Proof Let $q$ be any path in $M$. If $q$ contains $(i, j)$, then $q$ has two consecutive parts, say $q_{1}$ and $q_{2}$, such that $q_{1} \in \mathcal{P}\left(M_{1}\right)$ and $q_{2} \in \mathcal{P}\left(M_{2}\right)$. Hence, $p_{1} \prec q_{1}$ and $p_{2} \prec q_{2}$, so by Lemma $2.10(\mathrm{v}), p_{1} \circ p_{2} \prec q_{1} \circ q_{2}$.

Now let $p:=p_{1} \circ p_{2}$ and suppose $q$ does not contain $(i, j)$. Then, $q$ either contains some $\left(i, j^{\prime}\right)$ with $j^{\prime}<j$, or some $\left(i^{\prime}, j\right)$ with $i^{\prime}<i$. We show how to construct extensions of $p$ and $q$ that witness that $p$ dominates $q$ in the first case, and remark that the second case can be shown symmetrically. We illustrate this situation in Fig. 5.

Suppose that $q$ contains $\left(i, j^{\prime}\right)$ with $j^{\prime}<j$. We show that $p \prec q$. First, $q$ also contains some $\left(i^{\prime}, j\right)$, where $i^{\prime}>i$. Let $h_{1}$ be the index of $\left(i, j^{\prime}\right)$ in $q$, i.e., $q\left(h_{1}\right)=$ $\left(i, j^{\prime}\right)$, and $h_{2}$ denote the index of $\left(i^{\prime}, j\right)$ in $q$, i.e., $q\left(h_{2}\right)=\left(i^{\prime}, j\right)$. We derive the following sequences from $q$.

- We let $q_{1}:=q(1), \ldots, q\left(h_{1}\right)$ and $q_{1}^{+}:=q_{1} \circ\left(i, j^{\prime}+1\right), \ldots,(i, j)$.

- We let $q_{12}:=q\left(h_{1}\right), \ldots, q\left(h_{2}\right)$.

- We let $q_{2}:=q\left(h_{2}\right), \ldots, q(l(q))$ and $q_{2}^{+}:=(i, j),(i+1, j), \ldots,\left(i^{\prime}, j\right) \circ q_{2}$. 
Fig. 5 Situation in the proof of Lemma 3.7

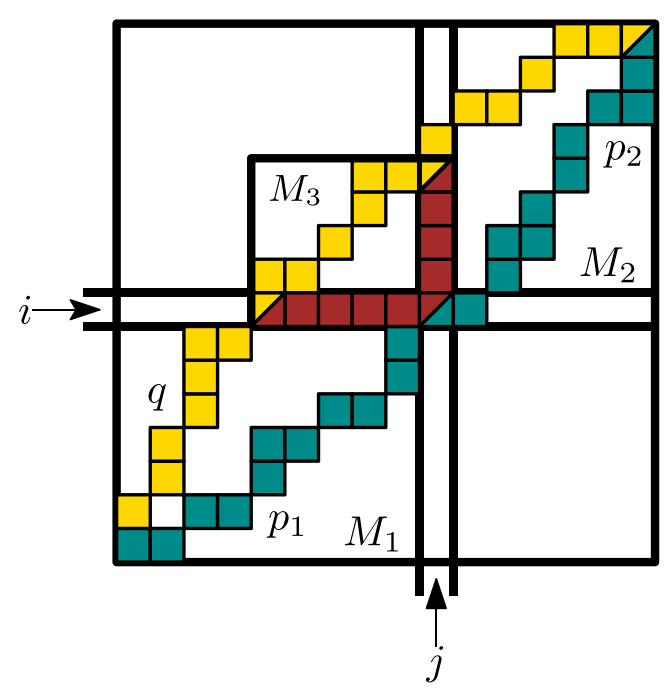

Since $q_{1}^{+} \in \mathcal{P}\left(M_{1}\right)$ and $p_{1} \prec \mathcal{P}\left(M_{1}\right)$, we have that $p_{1} \prec q_{1}^{+}$, similarly that $p_{2} \prec q_{2}^{+}$and considering $M_{3}:=M\left[i^{\prime} . . i, j . . j^{\prime}\right]$, we have by Lemma 3.6(i) that $p_{12}:=p_{\lrcorner}\left(M_{3}\right)=\left(i, j^{\prime}\right),\left(i, j^{\prime}+1\right), \ldots,(i, j),(i+1, j), \ldots,\left(i^{\prime}, j\right)$ dominates $q_{12}$.

Consequently, we consider the following extensions of these sequences.

(I) We let $e_{1} \in E\left(p_{1}\right)$ and $f_{1} \in E\left(q_{1}^{+}\right)$such that $l\left(e_{1}\right)=l\left(f_{1}\right)$ and $M\left[e_{1}\right] \leq$ $M\left[f_{1}\right]$.

(II) We let $e_{12} \in E\left(p_{12}\right)$, and $f_{12} \in E\left(q_{12}\right)$ such that $l\left(e_{12}\right)=l\left(f_{12}\right)$ and $M\left[e_{12}\right] \leq M\left[f_{12}\right]$.

(III) We let $e_{2} \in E\left(p_{2}\right)$, and $f_{2} \in E\left(q_{2}^{+}\right)$such that $l\left(e_{2}\right)=l\left(f_{2}\right)$ and $M\left[e_{2}\right] \leq$ $M\left[f_{2}\right]$.

We construct extensions $e^{\prime} \in E(p)$ and $f^{\prime} \in E(q)$. The idea of this construction is that we stretch $e_{1} \circ e_{2}$, the extensions of $p_{1}$ and $p_{2}$ to obtain $e^{\prime}$ and we stretch the extensions of the the three parts of $q$, namely $q_{1}, q_{12}$, and $q_{2}$, to obtain $f^{\prime}$ in such a way that the relations between these extensions can be used to guarantee that in the end, $M\left[e^{\prime}\right] \leq M\left[f^{\prime}\right]$. The most crucial step uses the fact that $q_{1}^{+}$shares a horizontal subpath with $p_{12}$ and that $q_{2}^{+}$shares a vertical subpath with $p_{12}$. Since $q_{1}^{+}$is a path in $M_{1}, p_{1} \prec q_{1}^{+}$, and since $p_{12} \prec q_{12}$ this allows for 'transferring' the domination property of $p_{1}$ over $q_{1}^{+}$to a part of $q_{12}$, via the subpath that $q_{1}^{+}$shares with $p_{12}$. Similar for the other part of $p_{12}$, using $p_{2}$.

Let $z$ be the last index in $q$ of any element that is matched up with $(i, j)$ in the extensions of (II). (Following the proof of Lemma 3.6, this would mean $z$ is the index of $\max \left(q_{12}\right)$ in $q$.) We first construct a pair of extensions $e_{j}^{\prime} \in E\left(p_{1}\right)$, and $f_{j}^{\prime} \in$ $E(q[1 . . z])$ with $l\left(e_{j}^{\prime}\right)=l\left(f_{j}^{\prime}\right)$ and $M\left[e_{j}^{\prime}\right] \leq M\left[f_{j}^{\prime}\right]$. With a symmetric procedure, we can obtain extensions of $p_{2}$ and of $q[(z+1) . . l(q)]$, and use them to obtain extensions of $p=p_{1} \circ p_{2}$ and $q=q[1 . . z] \circ q[(z+1) . . l(q)]$ witnessing that $p \prec q$. 
We give the details of the first part of the construction. Let $a$ be the index of the last repetition in $f_{1}$ of $q\left(h_{1}-1\right)$, i.e., the index that appears just before $q\left(h_{1}\right)=\left(i, j^{\prime}\right)$ in $f_{1}$. We let $e_{j^{\prime}-1}^{\prime}[1 . . a]:=e_{1}[1 . . a]$ and $f_{j^{\prime}-1}^{\prime}[1 . . a]:=f_{1}[1 . . a]$. By (I), $M\left[e_{j^{\prime}-1}^{\prime}\right] \leq$ $M\left[f_{j^{\prime}-1}^{\prime}\right]$.

For $x=j^{\prime}, j^{\prime}+1, \ldots, j$, we inductively construct $e_{x}^{\prime}$ and $f_{x}^{\prime}$ using $e_{x-1}^{\prime}$ and $f_{x-1}^{\prime}$, for an illustration see Fig. 6. We maintain as an invariant that $l\left(e_{x-1}^{\prime}\right)=l\left(f_{x-1}^{\prime}\right)$ and that $M\left[e_{x-1}^{\prime}\right] \leq M\left[f_{x-1}^{\prime}\right]$. Let $a_{1}, \ldots, a_{c}$ denote the indices of the occurrences of $(i, x)$ in $f_{1}$, and $b_{1}, \ldots, b_{d}$ denote the indices of the occurrences of $(i, x)$ in $e_{12}$. If $c=d$, meaning that $f_{1}$ and $e_{12}$ repeat $(i, x)$ the same number of times, then we can append $e_{1}\left[a_{1} . . a_{c}\right]$ to $e_{x-1}^{\prime}$ to obtain $e_{x}^{\prime}$ and $f_{12}\left[b_{1} . . b_{d}\right]$ to $f_{x-1}^{\prime}$ to obtain $f_{x}^{\prime}$. This way, we append the same number of elements to $e_{x-1}^{\prime}$ and to $f_{x-1}^{\prime}$; furthermore we know for each $\alpha \in\left[a_{1} . . a_{c}\right]$ and each $\beta \in\left[b_{1} . . b_{d}\right]$ that $M\left[e_{1}(\alpha)\right] \leq M[i, x] \leq M\left[f_{12}(\beta)\right]$ by the properties of the extensions that we use. Therefore, $M\left[e_{x}^{\prime}\right] \leq M\left[f_{x}^{\prime}\right]$.

If $c \neq d$, then we repeat the last element of the shorter one of $e_{1}\left[a_{1} . . a_{c}\right]$ and $f_{12}\left[b_{1} . . b_{d}\right]$ the corresponding number of times to obtain extensions of the same length. The argument that $M\left[e_{x}^{\prime}\right] \leq M\left[f_{x}^{\prime}\right]$ after this step is the same as the one outlined in the previous case. Formally, we let:

$$
\begin{array}{ll}
e_{x}^{\prime}:=e_{x-1}^{\prime} \circ e_{1}\left[a_{1} . . a_{c}\right] \text { and } f_{x}^{\prime}:=f_{x-1}^{\prime} \circ f_{12}\left[b_{1} . . b_{d}\right], & \text { if } c=d \\
e_{x}^{\prime}:=e_{x-1}^{\prime} \circ e_{1}\left[a_{1} . . a_{c}\right] \circ \overbrace{e_{1}\left(a_{c}\right), \ldots, e_{1}\left(a_{c}\right)}^{d-c \text { times }} \text { and } f_{x}^{\prime}:=\overbrace{f_{x-1}^{\prime} \circ f_{12}\left[b_{1} . . b_{d}\right],}^{f_{\text {times }}}, & \text { if } c<d \\
e_{x}^{\prime}:=e_{x-1}^{\prime} \circ e_{1}\left[a_{1} . . a_{c}\right] \text { and } f_{x}^{\prime}:=f_{x-1}^{\prime} \circ f_{12}\left[b_{1} . . b_{d}\right] \circ \overbrace{f_{12}\left(b_{d}\right), \ldots, f_{12}\left(b_{d}\right)}^{\prime}, & \text { if } c>d
\end{array}
$$

In each case, we extended $e_{x-1}^{\prime}$ and $f_{x-1}^{\prime}$ by the same number of elements; furthermore we know by (I) that for $y \in\left\{a_{1}, \ldots, a_{c}\right\}, M\left[e_{1}(y)\right] \leq M\left[f_{1}(y)\right]$, by choice we have that for all $y^{\prime} \in\left\{b_{1}, \ldots, b_{d}\right\}, f_{1}(y)=e_{12}\left(y^{\prime}\right)$ and we know that $M\left[e_{12}\left(y^{\prime}\right)\right] \leq M\left[f_{12}\left(y^{\prime}\right)\right]$ by (II). Hence, $M\left[e_{x}^{\prime}\right] \leq M\left[f_{x}^{\prime}\right]$ in either of the above cases. In the end of this process, we have $e_{j}^{\prime} \in E\left(p_{1}\right)$ and $f_{j}^{\prime} \in E(q[1 . . z])$, and by construction, $l\left(e_{j}^{\prime}\right)=l\left(f_{j}^{\prime}\right)$ and $M\left[e_{j}^{\prime}\right] \leq M\left[f_{j}^{\prime}\right]$.

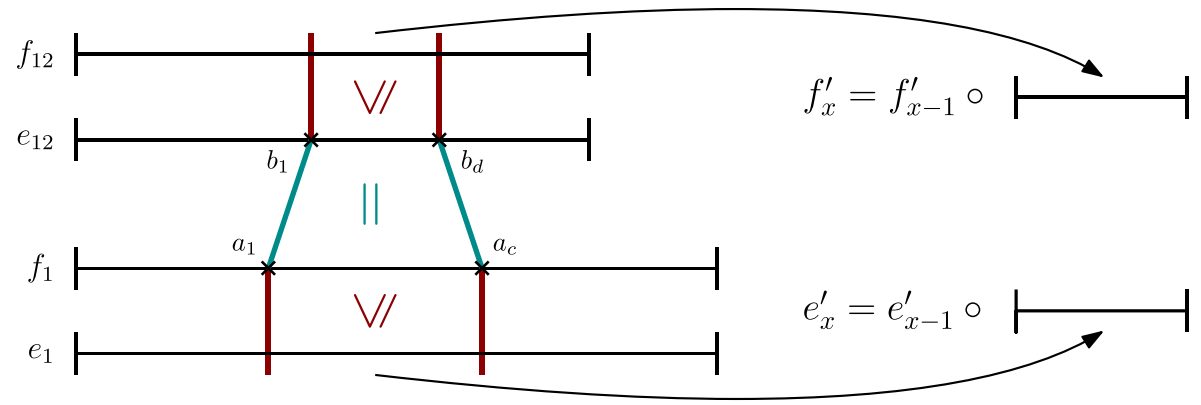

Fig. 6 Constructing extensions in the proof of Lemma 3.7 


\subsection{The Chop Lemmas}

Assume the notation of the Split Lemma. If we were to apply it recursively, it only yields a size reduction whenever $(i, j) \notin\{(1,1),(m, n)\}$. Motivated by this issue, we prove two more lemmas to deal with the cases when $(i, j) \in\{(1,1),(m, n)\}$, and we coin them the 'Chop Lemmas'. It will turn out that when applied to typical sequences, a repeated application of these lemmas yields a dominating path in $M$. This insight crucially helps in arguing that the dominating path in a merge matrix can be found using linearly many integer operations. Before we present their statements and proofs, we need another auxiliary lemma.

Lemma 3.8 Let $r$ and $c$ be two integer sequences of length 3 where for all $s \in\{r, c\}$,

$$
s(3) \leq s(1) \leq s(2) .
$$

Let $L$ be the merge matrix of $r$ and $c$. If $L[1,2] \leq L[2,1]$, then $p_{\lrcorner}(L) \prec p\ulcorner(L)$.

Proof This can be witnessed by the following extensions $e \in E\left(p_{\lrcorner}(L)\right)$ and $f \in$ $E(p\ulcorner(L))$ :

$$
\begin{aligned}
& e:(1,1),(1,2),(1,3),(2,3),(3,3),(3,3),(3,3) \\
& f:(1,1),(2,1),(2,1),(2,1),(3,1),(3,2),(3,3)
\end{aligned}
$$

We argue that $e \leq f$ :

$$
\begin{aligned}
L[1,2] \leq L[2,1] & \text { (by assumption) } \\
L[1,3] \leq L[1,2] \leq L[2,1] & (\text { since } c(3) \leq c(2) \text { and by assumption }) \\
L[2,3] \leq L[2,1] & (\text { since } c(3) \leq c(1)) \\
\forall i \in[3]: L[3,3] \leq L[3, i] & (\text { since } c(3) \leq c(1) \leq c(2))
\end{aligned}
$$

Remark 3.9 We would like to stress that up to this point, all results in this section were shown in terms of arbitrary integer sequences. For the next lemma, we require the sequences considered to be typical sequences. In Section 3.5 we will generalize the results that rely on the following lemmas to arbitrary integer sequences. The generalization to arbitrary integer sequences is necessary for the applications in Section 4, since the integer sequences arising there are in general not typical sequences.

We are now ready to prove the Chop Lemmas for typical sequences. They come in two versions, one that is suited for the case of the bottom left submatrix after an application of the Split Lemma to $M$, and one for the top right submatrix. In the former case, we have that the last row is a minimum row and that the last column is a minimum column. We will prove this lemma in more detail and observe that the other case follows by symmetry with the arguments given in the following proof. For an illustration of the setting in the following lemma, see Fig. $7 \mathrm{~b}$. 


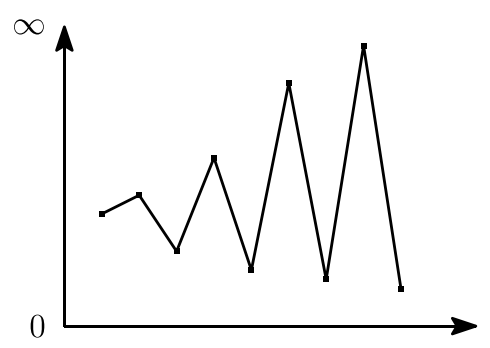

(a)

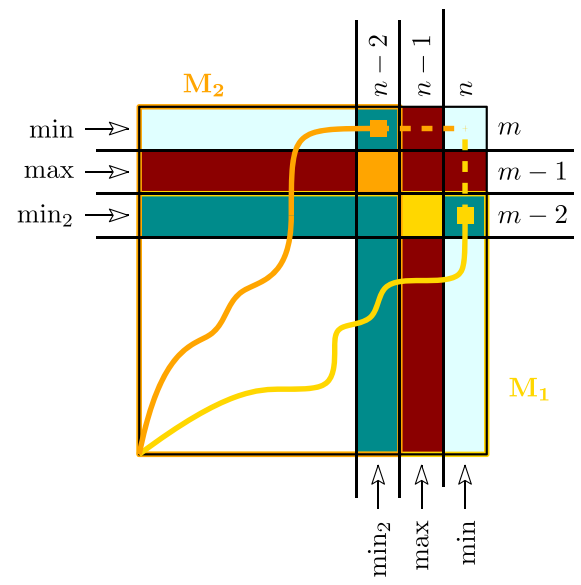

(b)

Fig. 7 Visual aides to the proof of Lemma 3.10. (a) Typical sequence ending in the minimum. (b) The general setting in Lemma 3.10

Lemma 3.10 (Chop Lemma - Bottom) Let $r$ and $c$ be typical sequences of length $m \geq 3$ and $n \geq 3$, respectively, and let $M$ be the merge matrix of $r$ and $c$. Suppose that $m \in \operatorname{argmin}(r)$ and $n \in \operatorname{argmin}(c)$ and let $M_{1}:=M[1 . .(m-2), 1 . . n]$ and $M_{2}:=M[1 . . m, 1 . .(n-2)]$ and for all $h \in[2]$, let $p_{h} \prec \mathcal{P}\left(M_{h}\right)$. Let $p_{1}^{+}:=p_{1} \circ$ $(m-1, n),(m, n)$ and $p_{2}^{+}:=p_{2} \circ(m, n-1),(m, n)$.

(i) If $M[m-2, n-1] \leq M[m-1, n-2]$, then $p_{1}^{+} \prec \mathcal{P}(M)$.

(ii) If $M[m-1, n-2] \leq M[m-2, n-1]$, then $p_{2}^{+} \prec(M)$.

Proof Let $s \in\{r, c\}$. Since $s$ is a typical sequence and $l(s) \in \operatorname{argmin}(s)$, we know by Corollary 2.8 that for all $k \in[\lfloor l(s) / 2\rfloor]$,

$l(s)-2 k+1 \in \operatorname{argmax}(s[1 . .(l(s)-2 k+1)])$ and $l(s)-2 k \in \operatorname{argmin}(s[1 . .(l(s)-2 k)])$.

Informally speaking, this means that the last element of $s$ is the minimum, the $(l(s)-1)$-th element of $s$ is the maximum, the $(l(s)-2)$-th element is 'secondsmallest' element, and so on. We will therefore refer to the element at position $l(s)-$ $2 k(2 k \leq l(s))$ as ' $\min _{k+1}(s)$ ' (note that the minimum is achieved when $k=0$, hence the ' +1 '), and elements at position $l(s)-2 k+1(2 k+1 \leq l(s)-1)$ as ' $\max _{k}(s)$ '. For an illustration of the shape of $s$ see Fig. 7a and for an illustration of the general setting of this proof see Fig. 7b. We prove (i) and remark that the argument for (ii) is symmetric.

First, we show that each path in $M$ is dominated by at least one of $p_{1}^{+}$and $p_{2}^{+}$.

Claim 3.10.1 Let $q \in \mathcal{P}(M)$. Then, for some $r \in[2], p_{r}^{+} \prec q$.

Proof We may assume that $q$ does not contain $(m-1, n-1)$ : if so, we could easily obtain a path $q^{\prime}$ from $q$ by some local replacements such that $q^{\prime}$ dominates $q$, since 
$M[m-1, n-1]$ is the maximum element of the matrix $M$. We may assume that $q$ either contains $(m-1, n)$ or $(m, n-1)$. Assume that the former holds, and note that an argument for the latter case can be given analogously. Since $q$ contains $(m-1, n)$, and since $q$ does not contain ( $m-1, n-1)$, we may assume that $q$ contains $(m-2, n)$ : if not, we can simply add $(m-2, n)$ before $(m-1, n)$ to obtain a path that dominates $q$ (recall that $n$ is the column indexed by the minimum of $c$ ). Now, let $\left.q\right|_{M_{1}}$ be the restriction of $q$ to $M_{1}$, we then have that $q=\left.q\right|_{M_{1}} \circ(m-1, n),(m, n)$. Since $p_{1}$ dominates all paths in $M_{1}$, it dominates $\left.q\right|_{M_{1}}$ and so $p_{1}^{+} \prec q$.

The remainder of the proof is devoted to showing that $p_{1}^{+}$dominates $p_{2}^{+}$which yields the lemma by Claim 3.10.1 and transitivity. To achieve that, we will show in a series of claims that we may assume that $p_{2}$ contains $(m-2, n-2)$. In particular, we show that if $p_{2}$ does not contain $(m-2, n-2)$, then there is another path in $M_{2}$ that does contain $(m-2, n-2)$ and dominates $p_{2}$.

Claim 3.10.2 We may assume that there is a unique $j \in[n-2]$ such that $p_{2}$ contains $(m-1, j)$.

Proof Clearly, $p_{2}$ has to pass through the row $m-1$ at some point. We show that we may assume that there is a unique such point. Suppose not and let $j_{1}, \ldots, j_{t}$ be such that $p_{2}$ contains all $\left(m-1, j_{i}\right)$, where $i \in[t]$. By the definition of a path in a matrix, we have that $j_{i+1}=j_{i}+1$ for all $i \in[t-1]$. Let $p_{2}^{\prime}$ be the path obtained from $p_{2}$ by replacing, for each $i \in[t-1]$, the element $\left(m-1, j_{i}\right)$ with the element $\left(m-2, j_{i}\right)$. Since $r(m-2) \leq r(m-1)$ (recall that $m-1 \in \operatorname{argmax}(r))$, it is not difficult to see that $p_{2}^{\prime}$ dominates $p_{2}$, and clearly, $p_{2}^{\prime}$ satisfies the condition of the claim.

Claim 3.10.3 Let $j \in[n-3]$ be such that $p_{2}$ contains $(m-1, j)$. If $j=n-2 k+1$ for some $k \in \mathbb{N}$ with $2 k+1 \leq n-1$, then there is a path $p_{2}^{\prime}$ that dominates $p_{2}$ and contains $(m-1, j+1)$.

Proof For an illustration see Fig. 8a. First, by Claim 3.10.2, we may assume that $j$ is unique. Moreover, since $j=n-2 k+1$ and $j+1=n-2 k+2=n-2(k-1)$,

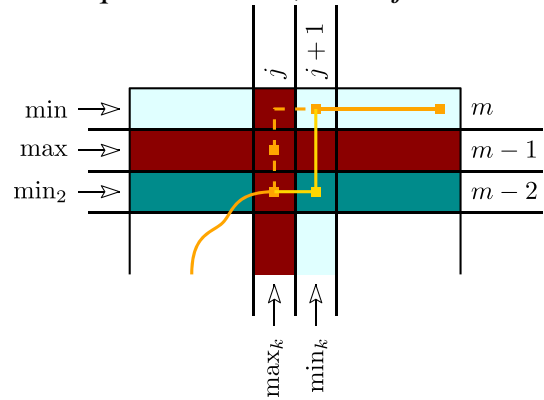

(a)

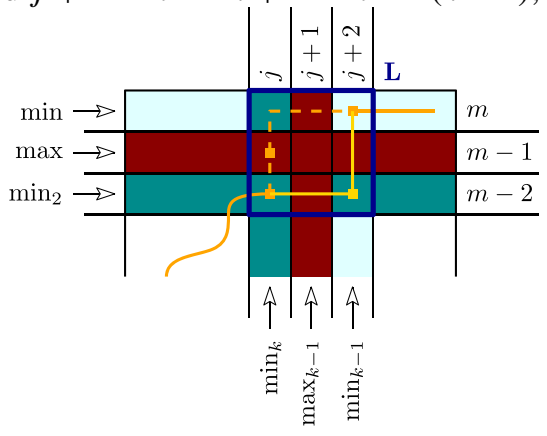

(b)

Fig. 8 Visualization of the arguments that lead to the conclusion that we may assume that $p_{2}$ contains $(m-2, n-2)$ in the proof of Lemma 3.10. (a) Situation of Claim 3.10.3. (b) Situation of Claim 3.10 .4 
we have that $c(j)=\max _{k}(c)$ and $c(j+1)=\min _{k}(c)$, respectively, and therefore $c(j+1) \leq c(j)$. Hence, we may assume that the element after $(m-1, j)$ in $p_{2}$ is $(m, j+1)$ : if $p_{2}$ contained $(m, j)$ we could simply remove $(m, j)$ from $p_{2}$ without changing the fact that $p_{2}$ is a dominating path since $M[m, j]>M[m, j+1]$. We modify $p_{2}$ as follows. We remove $(m-1, j)$, and add $(m-2, j)$ (if not already present), followed by $(m-2, j+1)$ and then $(m-1, j+1)$. For each $x \in\{M[m-2, j], M[m-2, j+1], M[m-1, j+1]\}$, we have that $x<M[m-1, j]$ (recall that $r(m-2)<r(m-1)$ and $c(j+1)<c(j))$. Hence, the resulting path dominates $p_{2}$ and it contains $(m-1, j+1)$.

Claim 3.10.4 Let $j \in[n-4]$ be such that $p_{2}$ contains $(m-1, j)$. If $j=n-2(k-1)$ for some $k \in\left[3 . .\left\lfloor\frac{n}{2}\right\rfloor\right]$, then there is a path $p_{2}^{\prime}$ that dominates $p_{2}$ and contains $(m-1, j+2)$.

Proof For an illustration see Fig. 8b. Again, by Claim 3.10.2, we may assume that $j$ is unique. Since $j=n-2(k-1)$, we have that $c(j)=\min _{k}(c)$. First, if not already present, we insert $(m-2, j)$ just before $(m-1, j)$ in $p_{2}$. This does not change the fact that $p_{2}$ is a dominating path, since $M[m-2, j]<M[m-1, j]$ (recall that $r(m-2)<$ $r(m-1))$. Next, consider the $3 \times 3$ submatrix $L:=M[(m-2) . . m, j . .(j+2)]$. Note that $L$ is the submatrix of $M$ restricted to the rows $\min (r), \max (r)$, and $\min _{2}(r)$, and the columns $\min _{k}(c), \max _{k-1}(c)$, and $\min _{k-1}(c)$. Furthermore, we may assume that $p_{2}$ restricted to $L$ is equal to $p\left\ulcorner(L)\right.$ : We know that $p_{2}$ contains $(m-2, j)$ and $(m-1, j)$, and with Claim 3.10.2, by which we can assume that $p_{2}$ contains no other element from row $m-1$, we can derive that the next element in $p_{2}$ is $(m, j)$ or $(m, j+1)$. In the latter case we can insert $(m, j)$ before $(m, j+1)$ since $M[m, j] \leq$ $M[m, j+1]$.

We show that $p_{\lrcorner}(L)$ dominates $p_{\ulcorner}(L)$, from which we can conclude that we can obtain a path $p_{2}^{\prime}$ from $p_{2}$ that contains $(m-1, j+2)$ and dominates $p_{2}$ by replacing $p\left\ulcorner(L)\right.$ with $p_{\lrcorner}(L)$. By Lemma 3.8, it suffices to show that $M[m-2, j+1] \leq$ $M[m-1, j]$, in other words, that $\max _{k-1}(c)+\min _{2}(r) \leq \max (r)+\min _{k}(c)$. hence,

By the assumption of the lemma, we have that $M[m-2, n-1] \leq M[m-1, n-2]$, $\max (c)+\min _{2}(r) \leq \max (r)+\min _{2}(c)$, and so: $\max (c)-\min _{2}(c) \leq \max (r)-\min _{2}(r)$.

Next, we have that for all $j \in[\lfloor n / 2\rfloor]$,

$$
\max (c)-\min _{2}(c) \geq \max _{j}(c)-\min _{j+1}(c) .
$$

Putting the two together, we have that

$\max _{k-1}(c)-\min _{k}(c) \leq \max (r)-\min _{2}(r)$, and so: $\max _{k-1}(c)+\min _{2}(r) \leq \max (r)+\min _{k}(c)$,

which concludes the proof of the claim.

We are now ready to conclude the proof.

Claim $3.10 .5 p_{1}^{+} \prec p_{2}^{+}$. 
Proof By repeated application of Claims 3.10.3 and 3.10.4, we know that there is a path $p_{2}^{\prime}$ in $M_{2}$ that contains $(m-1, n-2)$ and such that $p_{2}^{\prime} \prec p_{2}$. Furthermore, we may assume that $p_{2}^{\prime}$ contains $(m-2, n-2)$ as well: we can simply add this element if it is not already present; since $M[m-2, n-2] \leq M[m-1, n-2]$, this does not change the property that $p_{2}^{\prime} \prec p_{2}$. Now, let $p_{2}^{\prime \prime}$ be the subpath of $p_{2}^{\prime}$ ending in $(m-2, n-2)$. (Note that $p_{2}^{\prime \prime} \circ(m-2, n-1),(m-2, n) \in \mathcal{P}\left(M_{1}\right)$.) Then, the following hold:

$$
\begin{aligned}
p_{1}^{+} & \prec p_{2}^{\prime \prime} \circ(m-2, n-1),(m-2, n),(m-1, n),(m, n) \\
& \prec p_{2}^{\prime} \circ(m, n-1),(m, n) \\
& \prec p_{2}^{+}
\end{aligned}
$$

Here, (3) is due to $p_{1} \prec\left(M_{1}\right)$ and therefore $p_{1} \prec p_{2}^{\prime \prime} \circ(m-2, n-1),(m-2, n)$. Next, (5) is guaranteed since $p_{2}^{\prime} \prec p_{2}$. We justify (4) as follows: Let $L:=M[(m-$ 2)..m, $(n-2) . . n]$. Then, $p_{2}^{\prime \prime} \circ(m-2, n-1),(m-2, n),(m-1, n),(m, n)$ restricted to $L$ is $p_{\lrcorner}(L)$ and $p^{\prime} \circ(m, n-1),(m, n)$ restricted to $L$ is $p_{\ulcorner}(L)$. Since $L[1,2]=$ $M[m-2, n-1] \leq M[m-1, n-2]=L[2,1]$ by the assumption of this lemma (Lemma 3.10) we know that $p_{\lrcorner}(L) \prec p\ulcorner(L)$ by Lemma 3.8.

This concludes the proof of (i) and (ii) can be shown symmetrically.

As the previous lemma always assumes that $m \geq 3$ and $n \geq 3$, we observe the corresponding base case which occurs when either $m \leq 2$ or $n \leq 2$. This base case is justified by the observation that in the bottom case, the last row and column of $M$ are minimum.

Observation 3.11 (Base Case - Bottom) Let $r$ and $c$ be typical sequences of length $m$ and $n$, respectively, and let $M$ be the merge matrix of $r$ and $c$. Suppose that $m \in$ $\operatorname{argmin}(r)$ and $n \in \operatorname{argmin}(c)$. If $m \leq 2(n \leq 2)$, then ${ }^{5}$

$$
p^{*}:=(1,1),(m, 1),(m, 2), \ldots,(m, n) \quad\left(p^{*}:=(1,1),(1, n),(2, n), \ldots,(m, n)\right)
$$

dominates $\mathcal{P}(M)$, i.e., $p^{*} \prec \mathcal{P}(M)$.

By symmetry, we have the following consequence of Lemma 3.10.

Corollary 3.12 (Chop Lemma - Top) Let $r$ and $c$ be typical sequences of length $m \geq 3$ and $n \geq 3$, respectively, and let $M$ be the merge matrix of $r$ and $c$. Suppose that $1 \in \operatorname{argmin}(r)$ and $1 \in \operatorname{argmin}(c)$ and let $M_{1}:=M[3 . . m, 1 . . n]$ and $M_{2}:=$ $M[1 . . m, 3 . . n]$ and for all $h \in[2]$, let $p_{h} \prec \mathcal{P}\left(M_{h}\right)$. Let $p_{1}^{+}:=(1,1),(2,1) \circ p_{1}$ and $p_{2}^{+}:=(1,1),(1,2) \circ p_{2}$.

(i) If $M[3,2] \leq M[2,3]$, then $p_{1}^{+} \prec \mathcal{P}(M)$.

(ii) If $M[2,3] \leq M[3,2]$, then $p_{2}^{+} \prec \mathcal{P}(M)$.

\footnotetext{
${ }^{5}$ Note that in the following equation, if $m=1$, then strictly speaking we would have that $p^{*}$ repeats the element $(1,1)$ twice which is of course not our intention. For the sake of a clear presentation though, we will ignore this slight abuse of notation, also in similar instances throughout this section.
} 
Again, we observe the corresponding base case.

Observation 3.13 (Base Case - Top) Let $r$ and $c$ be typical sequences of length $m$ and $n$, respectively, and let $M$ be the merge matrix of $r$ and $c$. Suppose that $1 \in \operatorname{argmin}(r)$ and $1 \in \operatorname{argmin}(c)$. If $m \leq 2(n \leq 2)$, then

$$
p^{*}:=(1,1),(1,2), \ldots,(1, n),(m, n) \quad\left(p^{*}:=(1,1),(2,1), \ldots,(m, 1),(m, n)\right)
$$

dominates $\mathcal{P}(M)$, i.e., $p^{*} \prec \mathcal{P}(M)$.

\subsection{The Split-and-Chop Algorithm}

Equipped with the Split Lemma and the Chop Lemmas, we are now ready to give the algorithm that computes a dominating merge of two typical sequences. Consequently, we call this algorithm the 'Split-and-Chop Algorithm'.

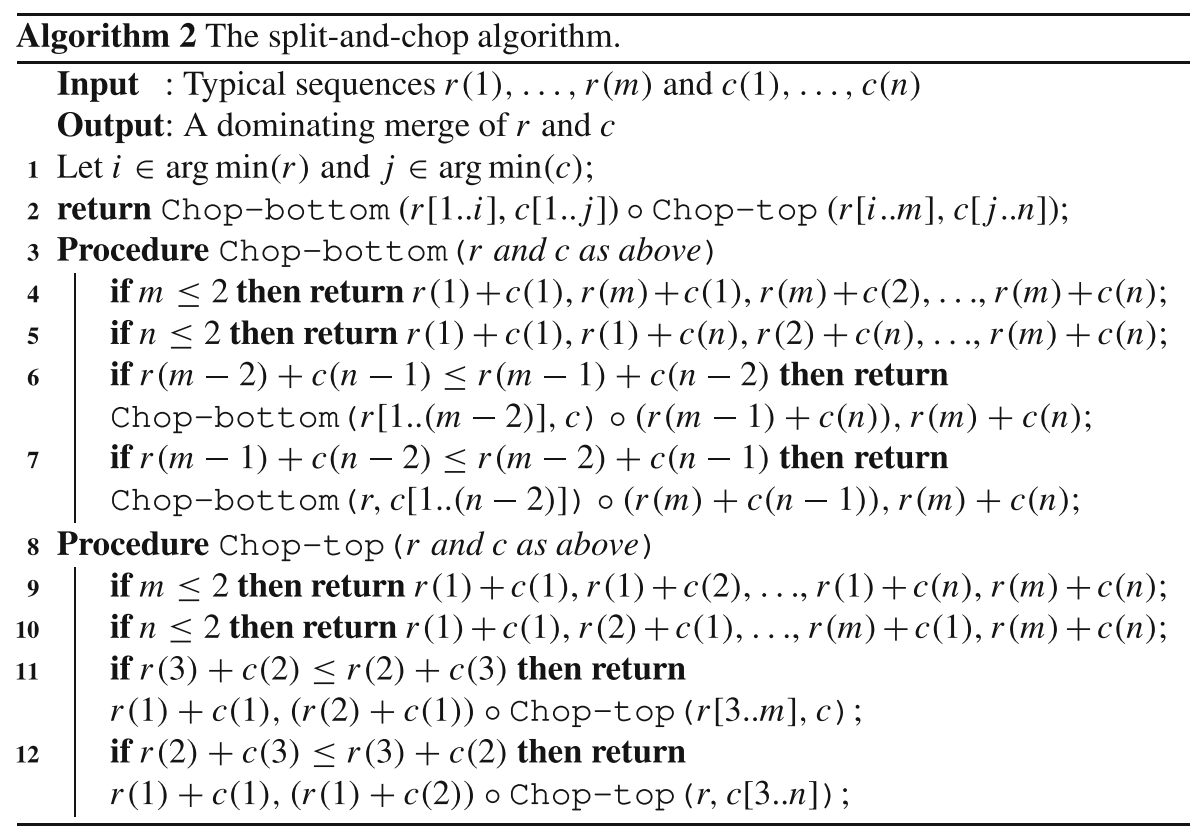

Lemma 3.14 Let $r$ and $c$ be typical sequences of length $m$ and $n$, respectively. Then, there is an algorithm that finds a dominating path in the merge matrix of $r$ and $c$ using $\mathcal{O}(m+n)$ integer operations.

Proof The algorithm practically derives itself from the Split Lemma (Lemma 3.7) and the Chop Lemmas (Lemma 3.10 and Corollary 3.12). However, to make the algorithm run in the claimed bound, we are not able to construct the merge matrix of $r$ and $c$. This turns out to be not necessary, as we can simply read off the crucial values upon which the recursion of the algorithm depends from the sequences directly. The details are given in Algorithm 2. 
The number of integer operations in the Chop-subroutines can be computed as $T(m+n) \leq T(m+n-2)+\mathcal{O}(1)$, which resolves to $\mathcal{O}(m+n)$. Correctness follows from Lemmas 3.7 and 3.10 and Corollary 3.12 with the base cases given in Observations 3.11 and 3.13.

\subsection{Generalization to Arbitrary Integer Sequences}

In this section we show how to generalize Lemma 3.14 to arbitrary integer sequences. In particular, we will show how to construct from a merge of two typical sequences $\tau(r)$ and $\tau(s)$ that dominates all of their merges, a merge of $r$ and $s$ that dominates all merges of $r$ and $s$. The claimed result then follows from an application of Lemma 3.14. We illustrate the following construction in Fig. 9.

The Typical Lift Let $r$ and $s$ be integer sequences and let $t \in \tau(r) \oplus \tau(s)$. Then, the typical lift of $t$, denoted by $\rho(t)$, is an integer sequence $\rho(t) \in r \oplus s$, obtained from $t$ as follows. For convenience, we will consider $\rho(t)$ as a path in the merge matrix $M$ of $r$ and $s$.

Step 1. We construct $t^{\prime} \in \tau(r) \boxplus \tau(s)$ such that $t^{\prime} \prec t$ using Lemma 3.5. Throughout the following, consider $t^{\prime}$ to be a path in the merge matrix $M_{\tau}$ of $\tau(r)$ and $\tau(s)$.

Step 2. First, we initialize $\rho_{t}^{1}:=t^{\prime}(1)=(1,1)$. For $i=\left\{2, \ldots, l\left(t^{\prime}\right)\right\}$, we proceed inductively as follows. Let $\left(i_{r}, i_{s}\right)=t(i)$ and let $\left(i_{r}^{\prime}, i_{s}^{\prime}\right)=t(i-1)$. (Note that $t(i-1)$ and $t(i)$ are indices in $M_{\tau}$.) Let furthermore $\left(j_{r}, j_{s}\right)$ be the index in $M$ corresponding to $\left(i_{r}, i_{s}\right)$, and let $\left(j_{r}^{\prime}, j_{s}^{\prime}\right)$ be the index in $M$ corresponding to $\left(i_{r}^{\prime}, i_{s}^{\prime}\right)$. Assume by induction that $\rho_{t}^{i-1} \in \mathcal{P}\left(M\left[1 . . j_{r}^{\prime}, 1 . . j_{s}^{\prime}\right]\right)$. We show how to extend $\rho_{t}^{i-1}$ to a path in $\rho_{t}^{i}$ in $M\left[1 . . j_{r}, 1 . . j_{s}\right]$. Since $t^{\prime}$ is non-diagonal, we have that $\left(i_{r}^{\prime}, i_{s}^{\prime}\right) \in\left\{\left(i_{r}-1, i_{s}\right),\left(i_{r}, i_{s}-1\right)\right\}$, so one of the two following cases applies.

Case S2.1 $\left(i_{r}^{\prime}=i_{r}-1\right.$ and $\left.i_{s}^{\prime}=i_{s}\right)$. In this case, we let $\rho_{t}^{i}:=\rho_{t}^{i-1}$ o $\left(j_{r}^{\prime}+1, j_{s}\right), \ldots,\left(j_{r}, j_{s}\right)$.

Case S2.2 $\left(i_{r}^{\prime}=i_{r}\right.$ and $\left.i_{s}^{\prime}=i_{s}-1\right)$. In this case, we let $\rho_{t}^{i}:=\rho_{t}^{i-1}$ o $\left(j_{r}, j_{s}^{\prime}+1\right), \ldots,\left(j_{r}, j_{s}\right)$.
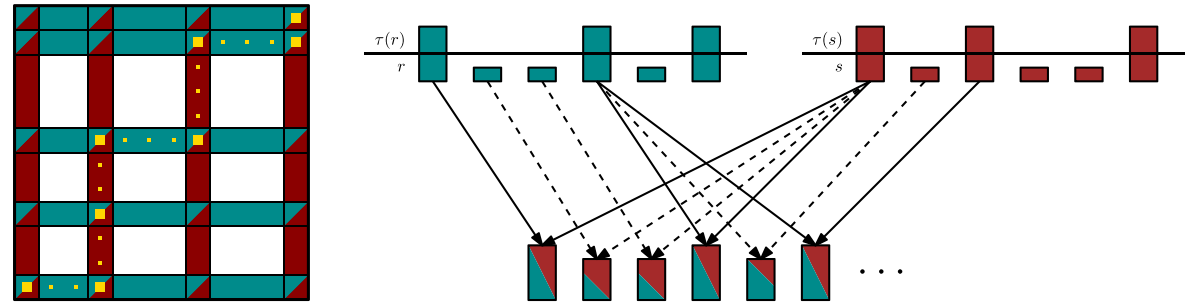

Fig. 9 Illustration of the typical lift. On the left side, the view of the merge matrix $M$, with the rows and columns corresponding to elements of the typical sequences highlighted. Inside there, $M_{\tau}$ can be seen as a highlighted submatrix. The merge $t^{\prime}$ is depicted as the large highlighted squares within $M_{\tau}$ and the small highlighted squares outside of $M_{\tau}$ show its completion to the typical lift of $t$. On the right side, an illustration that does not rely on the 'matrix view' 
Step 3. We return $\rho(t):=\rho_{t}^{l\left(t^{\prime}\right)}$.

Furthermore, it is readily seen that the typical lift contains no diagonal steps: we obtain it from a non-diagonal path in the merge matrix of $\tau(r)$ and $\tau(s)$ by inserting vertical and horizontal paths from the merge matrix of $r$ and $s$ between consecutive elements. Moreover, it is computable using linearly many integer operations, with Step 1 taking linearly many integer operations by Lemma 3.5. We summarize in the following observation.

Observation 3.15 Let $r$ and $s$ be integer sequences of length $m$ and $n$, respectively, and let $t \in \tau(r) \oplus \tau(s)$. Then, $\rho(t) \in r \boxplus s$, and $\rho(t)$ can be computed using $\mathcal{O}(m+n)$ integer operations.

We now show that if $t \in \tau(r) \oplus \tau(s)$ dominates all merges of $\tau(r)$ and $\tau(s)$, then the typical lift of $t$ dominates all merges of $r$ and $s$.

Lemma 3.16 Let $r$ and $s$ be integer sequences and let $q \in r \oplus s$. Let $t \in \tau(r) \oplus \tau(s)$ such that $t \prec \tau(r) \oplus \tau(s)$. Then, $\rho(t) \prec q$.

Proof Let $t^{\prime} \in \tau(r) \boxplus \tau(s)$ be the non-diagonal merge such that $t^{\prime} \prec t$ used in the construction of $\rho(t)$. We argue that $\rho(t) \prec t^{\prime}$. To see this, let $M$ be the merge matrix of $r$ and $s$ and consider any $\left(j_{r}^{\prime}, j_{s}^{\prime}\right)$ and $\left(j_{r}, j_{s}\right)$ as in Step 2, and suppose that $j_{s}^{\prime}=j_{s}$. (Note that either $j_{s}^{\prime}=j_{s}$ or $j_{r}^{\prime}=j_{r}$.) As the only elements of the typical sequence of $r$ in $\left[j_{r}^{\prime} . . j_{r}\right]$ are $r\left(j_{r}^{\prime}\right)$ and $r\left(j_{r}\right)$, we know that either for all $h_{r} \in\left[j_{r}^{\prime} . . j_{r}\right]$, $r\left(j_{r}^{\prime}\right) \leq r\left(h_{r}\right) \leq r\left(j_{r}\right)$, or for all $h_{r} \in\left[j_{r}^{\prime} . . j_{r}\right], r\left(j_{r}^{\prime}\right) \geq r\left(h_{r}\right) \geq r\left(j_{r}\right)$. Therefore, in an extension of $t^{\prime}$, we can repeat the index that yields $\max \left\{M\left[j_{r}^{\prime}, j_{s}\right], M\left[j_{r}, j_{s}\right]\right\}$ sufficiently many (i.e., $j_{r}-j_{r}^{\prime}$ ) times to ensure that the value of the extension of $t^{\prime}$ is an upper bound for all values of $\rho(t)$ in these positions.

To finish the proof, we have by Lemma 2.10(iii) that there exists a $q^{\prime} \in \tau(r) \oplus \tau(s)$ such that $q^{\prime} \prec q$. Since $t \prec \tau(r) \oplus \tau(s)$, we can conclude:

$$
\rho(t) \prec t^{\prime} \prec t \prec q^{\prime} \prec q .
$$

We wrap up and prove the Merge Dominator Lemma (Lemma 3.1), stated here in the slightly stronger form that the dominating merge is non-diagonal (which is necessary for the applications in Section 4).

Lemma 3.17 (Merge Dominator Lemma) Let $r$ and $c$ be integer sequence of length $m$ and $n$, respectively. There exists a dominating non-diagonal merge of $r$ and $c$, i.e., an integer sequence $t \in r \boxplus c$ such that $t \prec r \oplus c$, and this dominating merge can be computed using $\mathcal{O}(m+n)$ integer operations.

Proof The algorithm proceeds in the following steps.

Step 1. Compute $\tau(r)$ and $\tau(c)$.

Step 2. Apply the Split-and-Chop Algorithm on input $(\tau(r), \tau(c))$ to obtain $t \prec$ $\tau(r) \oplus \tau(c)$.

Step 3. Return the typical lift $\rho(t)$ of $t$. 
Correctness of the above algorithm follows from Corollary 3.4 and Lemmas 3.14 and 3.16 which together guarantee that $\rho(t) \prec r \oplus c$, and by Observation 3.15, $\rho(t)$ is a non-diagonal merge, i.e., $\rho(t) \in r \boxplus c$. By Lemma 2.9, Step 1 can be done using $\mathcal{O}(m+n)$ integer operations, by Lemma 3.14, Step 2 takes $\mathcal{O}(m+n)$ integer operations as well, and by Observation 3.15, the typical lift of $t$ can also be computed using $\mathcal{O}(m+n)$ integer operations. Hence, the overall number of integer operations needed is $\mathcal{O}(m+n)$.

\section{Directed Width Measures of Series Parallel Digraphs}

In this section, we give algorithmic consequences of the Merge Dominator Lemma. In Section 4.1, we provide a polynomial time algorithm that computes the (weighted) cutwidth of (arc-weighted) series parallel digraphs. In Section 4.2 we provide a linear time transformation that allows for computing the modified cutwidth of an SPD on $n$ vertices in polynomial time using the algorithm that computes the weighted cutwidth of an arc-weighted SPD.

\subsection{Cutwidth}

Recall that given a topological order $v_{1}, \ldots, v_{n}$ of a directed acyclic graph $G$, its cutwidth is the maximum over all $i \in[n-1]$ of the number of arcs that have their tail vertex in $\left\{v_{1}, \ldots, v_{i}\right\}$ and their head vertex in $\left\{v_{i+1}, \ldots, v_{n}\right\}$, and that the cutwidth of $G$ is the minimum cutwidth over all its topological orders.

To give the algorithm for the corresponding CUTWIDTH OF SERIES PARALLEL DIGRAPHS problem, we consider a generalized version where the input digraph has edge weights and we want to find a topological order that minimizes the weighted cutwidth.

Definition 4.1 Let $G$ be a directed acyclic graph and $\omega: A(G) \rightarrow \mathbb{N}$ be a weight function. ${ }^{6}$ For a topological order $\pi \in \Pi(G)$ of $G$, the weighted cutwidth of $(\pi, \omega)$ is defined as

$$
\operatorname{wcutw}(\pi, \omega):=\max _{i \in[n-1]} \sum_{\substack{(v, w) \in A(G) \\ \pi(v) \leq i, \pi(w)>i}} \omega(v, w),
$$

and the weighted cutwidth of $(G, \omega)$ is $\operatorname{wcutw}(G, \omega):=\min _{\pi \in \Pi(G)} \operatorname{wcutw}(\pi, \omega)$.

The corresponding computational problem is defined as follows.

Weighted CutwidTh of SERIES PARAllel DigraphS

Input: A series parallel digraph $G$ and an arc-weight function $\omega: A(G) \rightarrow \mathbb{N}$.

Question: What is the weighted cutwidth of $(G, \omega)$ ?

\footnotetext{
${ }^{6}$ For an $\operatorname{arc}(v, w)$, we use the shorthand ' $\omega(v, w)$ ' for ' $\omega((v, w))$ '.
} 
The Cutwidth of Series Parallel Digraphs problem is the special case of the Weighted Cutwidth of Series Parallel Digraphs problem where all arcs have weight 1 . Throughout this section, we refer to arc-weighted directed acyclic graphs simply as weighted directed acyclic graphs.

Given a weighted series parallel digraph $(G, \omega)$, our algorithm follows a bottomup dynamic programming scheme along the decomposition tree $T$ that yields $G$. Each node $t \in V(T)$ has a subgraph $G_{t}$ of $G$ associated with it, that is also series parallel. Naturally, we use the property that $G_{t}$ is obtained either via series or parallel composition of the SPD's associated with its two children.

To make this problem amenable to be solved using merges of integer sequences, we define the following notion of a cut-size sequence of a topological order of a directed acyclic graph which records for each position in the order, how many arcs cross it.

Definition 4.2 (Cut-Size Sequence) Let $(G, \omega)$ be a weighted directed acyclic graph on $n$ vertices and let $\pi \in \Pi(G)$ be a topological order of $G$. The sequence $x(1), \ldots, x(n-1)$, where for $i \in[n-1]$,

$$
x(i)=\sum_{\substack{(u, v) \in A(G) \\ \pi(u) \leq i, \pi(v)>i}} \omega(u, v)
$$

is the cut-size sequence of $\pi$, and denoted by $\sigma(\pi)$. For a set of topological orders $\Pi^{\prime} \subseteq \Pi(G)$, we let $\sigma\left(\Pi^{\prime}\right):=\left\{\sigma(\pi) \mid \pi \in \Pi^{\prime}\right\}$.

Throughout the remainder of this section, we slightly abuse notation: If $G_{1}$ and $G_{2}$ are SPD's that are being composed with a series composition, and $\pi_{1} \in \Pi\left(G_{1}\right)$ and $\pi_{2} \in \Pi\left(G_{2}\right)$, then we consider $\pi=\pi_{1} \circ \pi_{2}$ to be the concatenation of the two topological orders where $t_{2}=s_{1}$ only appears once in $\pi$. Moreover, to simplify notation, we consider the weight function of the given SPD only implicitly in places where it does not crucially influence the arguments.

We first argue via two simple observations that when computing the weighted cutwidth of a weighted series parallel digraph $G$ by following its decomposition tree in a bottom up manner, we only have to keep track of a set of topological orders that induce a set of cut-size sequences that dominate all cut-size sequences of $G$.

Observation 4.3 Let $G$ be a weighted DAG and $\pi, \lambda \in \Pi(G)$. If $\sigma(\pi) \prec \sigma(\lambda)$, then $\operatorname{wcutw}(\pi) \leq \operatorname{wcutw}(\lambda)$.

This is simply due to the fact that $\sigma(\pi) \prec \sigma(\lambda)$ implies that $\max (\sigma(\pi)) \leq$ $\max (\sigma(\lambda))$. Next, if $G$ is obtained from $G_{1}$ and $G_{2}$ via series or parallel composition, and we have $\pi_{1}, \lambda_{1} \in \Pi\left(G_{1}\right)$ such that $\sigma\left(\pi_{1}\right) \prec \sigma\left(\lambda_{1}\right)$, then it is always beneficial to choose $\pi_{1}$ over $\lambda_{1}$, and $\lambda_{1}$ can be disregarded.

Observation 4.4 Let $G$ be a weighted SPD that is obtained via series or parallel composition from weighted SPD's $G_{1}$ and $G_{2}$. Let $\pi_{1}, \lambda_{1} \in \Pi\left(G_{1}\right)$ be such that $\sigma\left(\pi_{1}\right) \prec \sigma\left(\lambda_{1}\right)$. Let $\pi, \lambda \in \Pi(G)$ be such that $\left.\pi\right|_{V\left(G_{1}\right)}=\pi_{1},\left.\lambda\right|_{V\left(G_{1}\right)}=\lambda_{1}$, and for all $v \in V\left(G_{2}\right), \pi(v)=\lambda(v)$. Then, $\sigma(\pi) \prec \sigma(\lambda)$. 
The previous observation is justified as follows. Let $\sigma(\lambda)=x(1), \ldots, x(n-1)$ and $\sigma(\lambda)=y(1), \ldots, y(n-1)$. Then, for each $i \in[n-1]$, the arcs of $G_{2}$ contribute equally to the values $x(i)$ and $y(i)$ (in particular since $G_{1}$ and $G_{2}$ are arc-disjoint). Therefore, we can use extensions of $\sigma\left(\pi_{1}\right)$ and $\sigma\left(\lambda_{1}\right)$ that witnesses that $\sigma\left(\pi_{1}\right) \prec$ $\sigma\left(\lambda_{1}\right)$ to construct extensions of $\sigma(\pi)$ and $\sigma(\lambda)$ that witness that $\sigma(\pi) \prec \sigma(\lambda)$.

The following lemma states that the cut-size sequences of a weighted SPD $G$ can be computed by pairwise concatenation or non-diagonal merging (depending on whether $G$ is obtained via series or parallel composition) of the two smaller SPD's that $G$ is obtained from. Intuitively speaking, the reason why we can only consider non-diagonal merges is the following. When $G$ is obtained from $G_{1}$ and $G_{2}$ via parallel composition, then each topological order of $G$ can be considered the 'merge' of a topological order of $G_{1}$ and one of $G_{2}$, where each position (apart from the first and the last) contains a vertex either from $G_{1}$ or from $G_{2}$. Now, in a merge of a cutsize sequence of $G_{1}$ with a cut-size sequence of $G_{2}$, a diagonal step would essentially mean that in some position, we insert both a vertex from $G_{1}$ and a vertex of $G_{2}$; this is of course not possible.

Lemma 4.5 Let $G_{1}$ and $G_{2}$ be weighted SPD's. Then the following hold.

$$
\begin{aligned}
& \sigma\left(\Pi\left(G_{1} \bullet G_{2}\right)\right)=\sigma\left(\Pi\left(G_{1}\right)\right) \odot \sigma\left(\Pi\left(G_{2}\right)\right) . \\
& \sigma\left(\Pi\left(G_{1} / / G_{2}\right)\right)=\sigma\left(\Pi\left(G_{1}\right)\right) \boxplus \sigma\left(\Pi\left(G_{2}\right)\right) .
\end{aligned}
$$

Proof (i). Let $\sigma(\pi) \in \sigma\left(\Pi\left(G_{1} \bullet G_{2}\right)\right)$ be such that $\pi$ is a topological order of $G_{1} \bullet G_{2}$. Then, $\pi$ consists of two contiguous parts, namely $\pi_{1}:=\left.\pi\right|_{V\left(G_{1}\right)} \in \Pi\left(G_{1}\right)$ followed by $\pi_{2}:=\left.\pi\right|_{V\left(G_{2}\right)} \in \Pi\left(G_{2}\right)$. Since there are no arcs from $V\left(G_{1}\right) \backslash\left\{t_{1}\right\}$ to $V\left(G_{2}\right) \backslash\left\{s_{2}\right\}$, we have that $\sigma(\pi)=\sigma\left(\pi_{1}\right) \circ \sigma\left(\pi_{2}\right) \in \sigma\left(\Pi\left(G_{1}\right)\right) \odot \sigma\left(\Pi\left(G_{2}\right)\right)$. The other inclusion follows similarly.

(ii). Let $\sigma(\pi) \in \sigma\left(\Pi\left(G_{1} / / G_{2}\right)\right)$ be such that $\pi$ is a topological order of $G_{1} / / G_{2}$. Let $\pi_{1}:=\left.\pi\right|_{V\left(G_{1}\right)}$ and $\pi_{2}:=\left.\pi\right|_{V\left(G_{2}\right)}$. It is clear that $\pi_{1} \in \Pi\left(G_{1}\right)$ and that $\pi_{2} \in$ $\Pi\left(G_{2}\right)$. Let $\sigma(\pi)=x(1), \ldots, x(n-1), \sigma\left(\pi_{1}\right)=y_{1}(1), \ldots, y_{1}\left(n_{1}-1\right)$, and $\sigma\left(\pi_{2}\right)=$ $y_{2}(1), \ldots, y_{2}\left(n_{2}-1\right)$. For any $i \in\{1, \ldots, n-1\}$, let $i_{1}$ be the maximum index such that $\pi\left(\pi_{1}^{-1}\left(i_{1}\right)\right) \leq i$, and define $i_{2}$ accordingly. Then, the set of arcs that cross the cut between positions $i$ and $i+1$ in $\pi$ is the union of the set of arcs crossing the cut between positions $i_{1}$ and $i_{1}+1$ in $\pi_{1}$ and the set of arcs crossing the cut between positions $i_{2}$ and $i_{2}+1$ in $\pi_{2}$. Since $G_{1}$ and $G_{2}$ are arc-disjoint, this means that $x(i)=y_{1}\left(i_{1}\right)+y_{2}\left(i_{2}\right)$. Together with the observation that each vertex at position $i+1<n$ in $\pi$ is either from $G_{1}$ or from $G_{2}$, we have that

$$
x(i+1) \in\left\{y_{1}\left(i_{1}+1\right)+y_{2}\left(i_{2}\right), y_{1}\left(i_{1}\right)+y_{2}\left(i_{2}+1\right)\right\},
$$

in other words, we have that $\sigma(\pi) \in \sigma\left(\pi_{1}\right) \boxplus \sigma\left(\pi_{2}\right) \subseteq \sigma\left(\Pi\left(G_{1}\right)\right) \boxplus \sigma\left(\Pi\left(G_{2}\right)\right)$. The other inclusion can be shown similarly, essentially using the fact that we are only considering non-diagonal merges.

We now prove the crucial lemma of this section which states that we can compute a dominating cut-size sequence of a weighted SPD $G$ from dominating cut-size sequences of the smaller weighted SPD's that $G$ is obtained from. For technical reasons, we assume in the following lemma that $G$ has no parallel arcs. 
Lemma 4.6 Let $G$ be a weighted SPD without parallel arcs. Then there is a topological order $\pi^{*}$ of $G$ such that $\sigma\left(\pi^{*}\right)$ dominates all cut-size sequences of $G$. Moreover, the following hold. Let $G_{1}$ and $G_{2}$ be weighted SPD's and for $r \in$ [2], let $\pi_{r}^{*}$ be a topological order of $G_{r}$ such that $\sigma\left(\pi_{r}^{*}\right)$ dominates all cut-size sequences of $G_{r}$.

(i) If $G=G_{1} \bullet G_{2}$, then $\pi^{*}=\pi_{1}^{*} \circ \pi_{2}^{*}$.

(ii) If $G=G_{1} / / G_{2}$, then $\pi^{*}$ can be found as the topological order of $G$ such that $\sigma\left(\pi^{*}\right)$ dominates $\sigma\left(\pi_{1}^{*}\right) \boxplus \sigma\left(\pi_{2}^{*}\right)$.

Proof We prove the lemma by induction on the number of vertices of $G$. If $|V(G)|=$ 2 , then the claim is trivially true (there is only one topological order). Suppose that $|V(G)|=: n>2$. Since $n>2$ and $G$ has no parallel arcs, we know that $G$ can be obtained from two SPD's $G_{1}$ and $G_{2}$ via series or parallel composition with $\left|V\left(G_{1}\right)\right|=: n_{1}<n$ and $\left|V\left(G_{2}\right)\right|=: n_{2}<n$. By the induction hypothesis, for $r \in[2]$, there is a unique topological order $\pi_{r}^{*}$ such that $\sigma\left(\pi_{r}^{*}\right)$ dominates all cut-size sequences of $G_{r}$.

Suppose $G=G_{1} \bullet G_{2}$. Since $\sigma\left(\pi_{1}^{*}\right)$ dominates all cut-size sequences of $G_{1}$ and $\sigma\left(\pi_{2}^{*}\right)$ dominates all cut-size sequences of $G_{2}$, we can conclude using Lemma 2.10(v) that $\sigma\left(\pi_{1}^{*}\right) \circ \sigma\left(\pi_{2}^{*}\right)$ dominates $\sigma\left(\Pi\left(G_{1}\right)\right) \odot \sigma\left(\Pi\left(G_{2}\right)\right)$ which together with Lemma 4.5(i) allows us to conclude that $\sigma\left(\pi_{1}^{*}\right) \circ \sigma\left(\pi_{2}^{*}\right)=\sigma\left(\pi_{1}^{*} \circ \pi_{2}^{*}\right)$ dominates all cut-size sequences of $G$. This proves (i).

Suppose that $G=G_{1} G_{2}$, and let $\pi^{*}$ be a topological order of $G$ such that $\sigma\left(\pi^{*}\right)$ dominates $\sigma\left(\pi_{1}^{*}\right) \boxplus \sigma\left(\pi_{2}^{*}\right)$. We show that $\sigma\left(\pi^{*}\right)$ dominates $\sigma(\Pi(G))$. Let $\pi \in \Pi(G)$. By Lemma 4.5(ii), there exist topological orders $\pi_{1} \in \Pi\left(G_{1}\right)$ and $\pi_{2} \in \Pi\left(G_{2}\right)$ such that $\sigma(\pi) \in \sigma\left(\pi_{1}\right) \boxplus \sigma\left(\pi_{2}\right)$. In other words, there are extensions $e_{1}$ of $\sigma\left(\pi_{1}\right)$ and $e_{2}$ of $\sigma\left(\pi_{2}\right)$ of the same length such that $\sigma(\pi)=e_{1}+e_{2}$. For $r \in$ [2], since $\sigma\left(\pi_{r}^{*}\right) \prec \sigma\left(\pi_{r}\right)$, we have that $\sigma\left(\pi_{r}^{*}\right) \prec e_{r}$. By Lemma 2.10(ii), ${ }^{7}$ there exists some $f \in \sigma\left(\pi_{1}^{*}\right) \oplus \sigma\left(\pi_{2}^{*}\right)$ such that $f \prec e_{1}+e_{2}$, and by Lemma 3.5, there is some $f^{\prime} \in \sigma\left(\pi_{1}^{*}\right) \boxplus \sigma\left(\pi_{2}^{*}\right)$ such that $f^{\prime} \prec f$. Since $\sigma\left(\pi^{*}\right) \prec \sigma\left(\pi_{1}^{*}\right) \boxplus \sigma\left(\pi_{2}^{*}\right)$, we have that $\sigma\left(\pi^{*}\right) \prec f^{\prime}$, and hence (ii) follows:

$$
\sigma\left(\pi^{*}\right) \prec f^{\prime} \prec f \prec e_{1}+e_{2}=\sigma(\pi) .
$$

We are now ready to prove the first main result of this section.

Theorem 4.7 Let $(G, \omega)$ be a weighted series parallel digraph on $n$ vertices and $m$ arcs, and let $W:=\sum_{(u, v) \in A(G)} \omega(u, v)$. There is an algorithm that computes in time $\mathcal{O}\left(\left(n^{2}+m\right) \log W\right)$ the weighted cutwidth of $(G, \omega)$, and outputs a topological order that achieves the upper bound.

Proof First, we modify $G$ so that it has no parallel arcs, without changing the weighted cutwidth. for any pair $u, v \in V(G)$ such that $G$ has $p>1$ parallel $(u, v)$-arcs, say $a_{1}, \ldots, a_{p}$, we replace $a_{1}, \ldots, a_{p}$ with a single arc $a^{*}$ of weight

\footnotetext{
${ }^{7}$ Take $r=e_{1}, s=e_{2}, r_{0}=\sigma\left(\pi_{1}\right)$, and $s_{0}=\sigma\left(\pi_{2}\right)$.
} 
$\sum_{i \in[p]} \omega\left(a_{i}\right)$. It is easy to see that this does not change the cutwidth, and clearly, the resulting graph is still series parallel. Moreover, this can be done in time at most $\mathcal{O}(m \log W)$, and we may from now on assume that $|A(G)|=\mathcal{O}\left(n^{2}\right)$.

We use the algorithm of Valdes et al. [25] to compute in time $\mathcal{O}(n+|A(G)|)=$ $\mathcal{O}\left(n^{2}\right)$ a decomposition tree $T$ that yields $G$, see Theorem 2.13. We process $T$ in a bottom-up fashion, and at each node $t \in V(T)$, compute a topological order $\pi_{t}$ of $G_{t}$, the series parallel digraph associated with node $t$, such that $\sigma\left(\pi_{t}\right)$ dominates all cut-size sequences of $G_{t}$. Let $t \in V(T)$.

Case 1 ( $t$ is a leaf node). In this case, $G_{t}$ is a single arc and there is precisely one topological order of $G_{t}$; we return that order.

Case 2 ( $t$ is a series node with left child $\ell$ and right child $r$ ). In this case, we look up $\pi_{\ell}$, a topological order such that $\sigma\left(\pi_{\ell}\right)$ dominates all cut-size sequences of $G_{\ell}$, and $\pi_{r}$, a topological order such that $\sigma\left(\pi_{r}\right)$ dominates all cut-size sequences of $G_{r}$. Following Lemma 4.6(i), we return $\pi_{\ell} \circ \pi_{r}$.

Case 3 ( $t$ is a parallel node with left child $\ell$ and right child $r$ ). In this case, we look up $\pi_{\ell}$ and $\pi_{r}$ as in Case 2, and we compute $\pi_{t}$ such that $\sigma\left(\pi_{t}\right)$ dominates $\sigma\left(\pi_{\ell}\right) \boxplus \sigma\left(\pi_{r}\right)$ using the Merge Dominator Lemma (Lemma 3.17). Following Lemma 4.6(ii), we return $\pi_{t}$.

Finally, we return $\pi_{\mathfrak{r}}$, the topological order (of $G_{\mathfrak{r}}=G$ ) computed for $\mathfrak{r}$, the root of $T$. Observations 4.3 and 4.4 ensure that it is sufficient to compute in each of the above cases a set $\Pi_{t}^{*} \subseteq \Pi\left(G_{t}\right)$ with the following property. For each $\pi_{t} \in \Pi\left(G_{t}\right)$, there is a $\pi_{t}^{*} \in \Pi_{t}^{*}$ such that $\sigma\left(\pi_{t}^{*}\right) \prec \sigma\left(\pi_{t}\right)$. By Lemma 4.6, we know that we can always find such a set of size one which is precisely what we compute in each of the above cases. Correctness of the algorithm follows. Since $T$ has $\mathcal{O}(n)$ nodes and each of the above cases can be handled in at most $\mathcal{O}(n)$ integer operations by Lemma 3.17, the total runtime of the algorithm after removing parallel arcs is $\mathcal{O}\left(n^{2} \log W\right)$, since the maximum value of any element in a cut-size sequence is trivially upper bounded by $W$. Therefore, the total runtime is $\mathcal{O}\left(\left(n^{2}+m\right) \log W\right)$.

We can easily use the algorithm of the previous theorem to solve the (unweighted) Cutwidth of Series Parallel Digraphs problem.

Corollary 4.8 Let $G$ be series parallel digraph on $n$ vertices and $m$ arcs. There is an algorithm that computes in time $\mathcal{O}\left(\left(n^{2}+m\right) \log m\right)$ the cutwidth of $G$, and outputs a topological order that achieves the upper bound.

Proof We create a weighted SPD $\left(G^{\prime}, \omega^{\prime}\right)$ as follows. The SPD $G^{\prime}$ is obtained from $G$ by replacing each set of parallel arcs from one vertex to another with a single arc. We let $\omega^{\prime}: A\left(G^{\prime}\right) \rightarrow \mathbb{N}$, such that for all $(u, v) \in A\left(G^{\prime}\right), \omega^{\prime}(u, v)$ is the number of parallel $(u, v)$-arcs in $G$. It is clear that the weighted cutwidth of $\left(G^{\prime}, \omega^{\prime}\right)$ is equal to the cutwidth of $G$. We can therefore apply the algorithm of Theorem 4.7 to find the cutwidth of $G$ via the weighted cutwidth of $\left(G^{\prime}, \omega^{\prime}\right)$. 


\subsection{Modified Cutwidth}

We now show how to use the algorithm for computing the weighted cutwidth of series parallel digraphs from Theorem 4.7 to give an algorithm that computes the modified cutwidth of a series parallel digraph in polynomial time. Recall that given a topological order $v_{1}, \ldots, v_{n}$ of a directed acyclic graph $G$, its modified cutwidth is the maximum over all $i \in[n-1]$ of the number of arcs that have their tail vertex in $\left\{v_{1}, \ldots, v_{i-1}\right\}$ and their head vertex in $\left\{v_{i+1}, \ldots, v_{n}\right\}$, and that the modified cutwidth of $G$ is the minimum modified cutwidth over all its topological orders. We are dealing with the following computational problem.

\section{Modified Cutwidth of SeRIEs PaRAllel DigRaphS}

Input: $\quad$ A series parallel digraph $G$.

Question: What is the modified cutwidth of $G$ ?

To solve this problem, we will provide a transformation which allows for applying the algorithm for the WEIGHTED CUTWIDTH OF SPD's problem to compute the modified cutwidth. We would like to remark that this transformation is similar to one provided in [6], however some modifications are necessary to ensure that the digraph resulting from the transformation is an SPD.

Theorem 4.9 Let $G$ be a series parallel digraph on $n$ vertices and $m$ arcs. There is an algorithm that computes in time $\mathcal{O}\left((n+m)^{2} \log m\right)$ the modified cutwidth of $G$, and outputs a topological order of $G$ that achieves the upper bound.

Proof We give a transformation that enables us to solve ModIFIED CUTWIDTH OF SPD's with help of an algorithm that solves WEIGHTED CUTWIDTH OF SPD's.

Let $\left(G^{\prime \prime},(s, t)\right)$ be an SPD on $n$ vertices and $m$ arcs. If $G^{\prime \prime}$ has parallel arcs then we subdivide all but one of the parallel arcs for each pair of vertices. This does not change the (modified) cutwidth, and keeps a digraph series parallel. Let $(G,(s, t))$ denote the resulting SPD which will be the input graph to the MODIFIED CUTWIDTH OF SPD's problem that we are solving. Note that $|V(G)|=\mathcal{O}(n+m)$ and that $|A(G)|=\mathcal{O}(m)$. We construct another digraph $G^{\prime}$ and an arc-weight function $\omega: A\left(G^{\prime}\right) \rightarrow \mathbb{N}$ as follows. For each vertex $v \in V(G) \backslash\{s, t\}$, we add to $G^{\prime}$ two vertices $v_{\text {in }}$ and $v_{\text {out }}$. We add $s$ and $t$ to $G^{\prime}$ and write $s$ as $s_{\text {out }}$ and $t$ as $t_{\text {in }}$. We add the following arcs to $G^{\prime}$. First, for each $v \in V(G)$, we add an $\operatorname{arc}\left(v_{\text {in }}, v_{\text {out }}\right)$ and we let $\omega\left(v_{\text {in }}, v_{\text {out }}\right):=$ $m+1$. Next, for each $\operatorname{arc}(v, w) \in A(G)$, we add an $\operatorname{arc}\left(v_{\text {out }}, w_{\text {in }}\right)$ to $G^{\prime}$ and we let $\omega\left(v_{\text {out }}, w_{\text {in }}\right):=1$. For an illustration see Fig. 10.
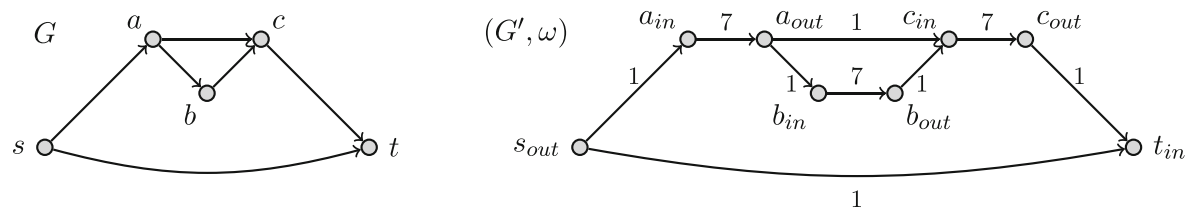

Fig. 10 Illustration of the transformation given in the proof of Theorem 4.9. Note that in this case, $m=6$, so the arcs between vertices $v_{\text {in }}$ and $v_{\text {out }}$ have weight 7 
We observe that the size of $G^{\prime}$ is linear in the size of $G$, and then prove that if $G^{\prime}$ is obtained from applying the above transformation to a series parallel digraph, then $G^{\prime}$ is itself an SPD.

Observation 4.9.1 Let $G$ and $G^{\prime}$ be as above. Then, $n^{\prime}:=\left|V\left(G^{\prime}\right)\right| \leq 2|V(G)|$ and $\left|A\left(G^{\prime}\right)\right| \leq|A(G)|+|V(G)|$.

Claim 4.9.2 If $G$ is a series parallel digraph, then $G^{\prime}$ as constructed above is an SPD.

Proof We prove the claim by induction on $n$, the number of vertices of $G$. For the base case when $n=2$, we have that $G$ is a single arc in which case $G^{\prime}$ is a single arc as well. Now suppose $n>2$. Since $n>2, G$ is obtained from two series parallel digraphs $G_{1}$ and $G_{2}$ via series or parallel composition. Since $G$ has no parallel arcs, we can use the induction hypothesis to conclude that the graphs $G_{1}^{\prime}$ and $G_{2}^{\prime}$ obtained via our construction are series parallel. Now, if $G=G_{1} / / G_{2}$, then it is immediate that $G^{\prime}$ is series parallel. If $G=G_{1} \bullet G_{2}$, then we have that in $G^{\prime}$, the vertex that was constructed since $t_{1}$ and $s_{2}$ were identified, call this vertex $x$, got split into two vertices $x_{i n}$ and $x_{\text {out }}$ with a directed arc of weight $m+1$ pointing from $x_{\text {in }}$ to $x_{\text {out }}$. Call the series parallel digraph consisting only of this arc $\left(X,\left(x_{\text {in }}, x_{\text {out }}\right)\right)$. We now have that $G^{\prime}=G_{1}^{\prime} \bullet X \bullet G_{2}^{\prime}$, so $G^{\prime}$ is series parallel in this case as well.

We are now ready to prove the correctness of this transformation. To do so, we will assume that we are given an integer $k$ and we want to decide whether the modified cutwidth of $G$ is at most $k$.

Claim 4.9.3 If $G$ has modified cutwidth at most $k$, then $G^{\prime}$ has weighted cutwidth at most $m+k+1$.

Proof Take a topological order $\pi$ of $G$ such that $\operatorname{mcutw}(\pi) \leq k$. We obtain $\pi^{\prime}$ from $\pi$ by replacing each vertex $v \in V(G) \backslash\{s, t\}$ by $v_{\text {in }}$ followed directly by $v_{\text {out }}$. Clearly, this is a topological order of $G^{\prime}$. We show that the weighted cutwidth of this order is at most $m+k+1$.

Let $i \in\left[n^{\prime}-1\right]$ and consider the cut between position $i$ and $i+1$ in $\pi^{\prime}$. We have to consider two cases. In the first case, there is some $v \in V(G)$ such that $\pi^{\prime-1}(i)=v_{\text {in }}$ and $\pi^{\prime-1}(i+1)=v_{\text {out }}$. Then, there is an arc of weight $m+1$ from $v_{\text {in }}$ to $v_{\text {out }}$ crossing this cut, and some other arcs of the form $\left(u_{\text {out }}, w_{\text {in }}\right)$ for some arc $(u, w) \in A(G)$. All these arcs cross position $\pi(v)$ in $\pi$, so since $\operatorname{mcutw}(\pi) \leq k$, there are at most $k$ of them. Furthermore, for each such arc we have that $\omega\left(\left(u_{\text {out }}, w_{\text {in }}\right)\right)=1$ by construction, so the total weight of this cut is at most $m+k+1$.

In the second case, we have that $\pi^{\prime-1}(i)=v_{\text {out }}$ and $\pi^{\prime-1}(i+1)=w_{\text {in }}$ for some $v, w \in V(G), v \neq w$. By construction, we have that $\pi(w)=\pi(v)+1$. Hence, any arc crossing the cut between $i$ and $i+1$ in $\pi^{\prime}$ is of one of the following forms.

(i) It is $\left(x_{\text {out }}, y_{i n}\right)$ for some $(x, y) \in A(G)$ with $\pi(x)<\pi(v)$ and $\pi(y)>\pi(v)$, or 
(ii) it is $\left(x_{\text {out }}, y_{\text {in }}\right)$ for some $(x, y) \in A(G)$ with $\pi(x)<\pi(w)$ and $\pi(y)>\pi(w)$, or

(iii) it is $\left(v_{\text {out }}, w_{\text {in }}\right)$.

Since $\operatorname{mcutw}(G) \leq k$, there are at most $k$ arcs of the first and second type, and since $G$ has no parallel arcs, there is at most one arc of the third type. By construction, all these arcs have weight one, so the total weight of this cut is $2 k+1 \leq m+k+1$.

Claim 4.9.4 If $G^{\prime}$ has weighted cutwidth at most $m+k+1$, then $G$ has modified cutwidth at most $k$.

Proof Let $\pi^{\prime}$ be a topological order of $G^{\prime}$ such that $\operatorname{wcutw}\left(\pi^{\prime}, \omega\right) \leq m+k+1$. First, we claim that for all $v \in V(G) \backslash\{s, t\}$, we have that $\pi^{\prime}\left(v_{\text {out }}\right)=\pi^{\prime}\left(v_{\text {in }}\right)+1$. Suppose not, for some vertex $v$. If we have that $\pi^{\prime}\left(v_{\text {in }}\right)<\pi^{\prime}\left(w_{\text {in }}\right)<\pi^{\prime}\left(v_{\text {out }}\right)$ for some $w \in V(G) \backslash\{s, t\}$ and $w \neq v$, then the cut between $\pi^{\prime}\left(w_{i n}\right)$ and $\pi^{\prime}\left(w_{i n}\right)+1$ has weight at least $2 m+2$ : the two arcs $\left(v_{\text {in }}, v_{\text {out }}\right)$ and $\left(w_{\text {in }}, w_{\text {out }}\right)$ cross this cut, and they are of weight $m+1$ each. Similarly, if $\pi^{\prime}\left(v_{\text {in }}\right)<\pi^{\prime}\left(w_{\text {out }}\right)<\pi^{\prime}\left(v_{\text {out }}\right)$, then the cut between $\pi^{\prime}\left(w_{\text {out }}\right)-1$ and $\pi^{\prime}\left(w_{\text {out }}\right)$ has weight at least $2 m+2$. Since $2 m+2>m+k+1$, we have a contradiction in both cases.

We define a linear order $\pi$ of $G$ as follows. We let $\pi(s):=1, \pi(t):=n$, and for all $v, w \in V(G) \backslash\{s, t\}$, we have $\pi(v)<\pi(w)$ if and only if $\pi^{\prime}\left(v_{i n}\right)<\pi^{\prime}\left(w_{i n}\right)$. It is clear that $\pi$ is a topological order of $G$; we show that $\pi$ has modified cutwidth at most $k$. Consider an arc $(x, y)$ that crosses a vertex $v$ in $\pi$, i.e., we have that $\pi(x)<\pi(v)<\pi(y)$. We have just argued that $\pi^{\prime}\left(v_{\text {out }}\right)=\pi^{\prime}\left(v_{i n}\right)+1$, so we have that the arc $\left(x_{\text {out }}, y_{\text {in }}\right)$ crosses the cut between $v_{\text {in }}$ and $v_{\text {out }}$ in $\pi^{\prime}$. Recall that there is an arc of weight $m+1$ from $v_{\text {in }}$ to $v_{\text {out }}$, so since $\operatorname{wcutw}\left(\pi^{\prime}, \omega\right) \leq m+k+1$, we can conclude that in $\pi$, there are at most $(m+k+1)-(m-1)=k \operatorname{arcs}$ crossing the vertex $v$ in $\pi$.

Now, to compute the modified cutwidth of $G$, we run the above described transformation to obtain $\left(G^{\prime}, \omega\right)$, and compute a topological order that gives the smallest weighted cutwidth of $\left(G^{\prime}, \omega\right)$ using Theorem 4.7. We can then follow the argument given in the proof of Claim 4.9.4 to obtain a topological order for $G$ that gives the smalles modified cutwidth of $G$.

By Claim 4.9.2, $G^{\prime}$ is an SPD, so we can indeed apply the algorithm of Theorem 4.7 to solve the instance $\left(G^{\prime}, \omega\right)$. Correctness follows from Claims 4.9.3 and 4.9.4. By Observation 4.9.1, $\left|V\left(G^{\prime}\right)\right|=\mathcal{O}(|V(G)|)=\mathcal{O}(n+m)$, and $\left|A\left(G^{\prime}\right)\right| \leq$ $|V(G)|+|A(G)|=\mathcal{O}(m+n)$, and clearly, $\left(G^{\prime}, \omega\right)$ can be constructed in time $\mathcal{O}(|V(G)|+|A(G)|)=\mathcal{O}(n+m)$; since $\sum_{(u, v) \in A\left(G^{\prime}\right)} \omega(u, v)=m^{\mathcal{O}(1)}$, the overall runtime of this procedure is at most $\mathcal{O}\left((n+m)^{2} \log m\right)$.

\section{Conclusions}

In this paper, we obtained a new technical insight in a now over a quarter century old technique, namely the use of typical sequences. The insight led to new polynomial 
time algorithms. Since its inception, algorithms based on typical sequences give the best asymptotic bounds for linear time FPT algorithms for treewidth and pathwidth, as functions of the target parameter. It still remains a challenge to improve upon these bounds $\left(2^{O\left(p w^{2}\right)}\right.$, respectively $\left.2^{O\left(t w^{3}\right)}\right)$, or give non-trivial lower bounds for parameterized pathwidth or treewidth. Possibly, the Merge Dominator Lemma can be helpful to get some progress here.

As other open problems, we ask whether there are other width parameters for which the Merge Dominator Lemma implies polynomial time or XP algorithms, or whether such algorithms exist for other classes of graphs. For instance, for which width measures can we give XP algorithms when parameterized by the treewidth of the input graph?

Lastly, we present one more open problem regarding the computation of width measures of series parallel digraphs. The vertex separation number of a topological order is the maximum over all cuts induced by the order of the number of vertices on the left side that have a neighbor on the right side. Finding a topological order that minimizes the vertex separation number corresponds to an important problem in compiler optimization, specifically to a problem related to register allocation: we are given a set of expressions (a "basic block" or "straight-line code") that have certain dependencies among each other and the task is to find a sequence for executing these expressions, respecting the dependencies, such that the number of used registers is minimized. The dependencies among these expressions form an acyclic digraph and any allowed schedule is a topological order. This problem was shown to be NP-hard by Sethi [20] while Kessler [15] gave a $2^{\mathcal{O}(n)}$ time exact algorithm, improving over the $n^{\mathcal{O}(n)}$ naive brute-force approach. Sethi and Ullman [21] showed in 1970 that the problem is linear time solvable if the acyclic digraph is a tree which (to the best of our knowledge) is the only known polynomial time case. It seems that with the help of the Merge Dominator Lemma, we might be able to obtain a polynomial time algorithm for this problem on series parallel digraphs. However, the application cannot be as immediate as in the case of cutwidth and modified cutwidth. The vertex separation number counts vertices rather than edges (as it is the case for cutwidth and modified cutwidth), and in a parallel composition, the sources of two SPDs are being identified. In a straightforward approach, this results in overcounting the contribution of the source vertex to several cuts, which is the main obstacle that needs to be overcome. While at first glance this may look like an issue that could be solved with rather straightforward techniques, we want to point out that (in our own experience) such direct approaches are fraught with very subtle pitfalls.

Acknowledgments We would like to thank anonymous reviewers for many helpful comments that greatly improved the presentation of the paper.

Funding Open access funding provided by University of Bergen (incl Haukeland University Hospital).

Open Access This article is licensed under a Creative Commons Attribution 4.0 International License, which permits use, sharing, adaptation, distribution and reproduction in any medium or format, as long as you give appropriate credit to the original author(s) and the source, provide a link to the Creative Commons 
licence, and indicate if changes were made. The images or other third party material in this article are included in the article's Creative Commons licence, unless indicated otherwise in a credit line to the material. If material is not included in the article's Creative Commons licence and your intended use is not permitted by statutory regulation or exceeds the permitted use, you will need to obtain permission directly from the copyright holder. To view a copy of this licence, visit http://creativecommonshorg/licenses/by/4. $0 /$.

\section{References}

1. Althaus, E., Ziegler, S.: Optimal tree decompositions revisited: a simpler linear-time FPT algorithm, CoRR, 1912.09144 (2019)

2. Amir, E.: Approximation algorithms for treewidth. Algorithmica 56(4), 448-479 (2010)

3. Bodlaender, H.L.: A linear-time algorithm for finding tree-decompositions of small treewidth. SIAM J. Comput. 25(6), 1305-1317 (1996)

4. Bodlaender, H.L., Cai, L., Chen, J., Fellows, M.R., Telle, J.A., Marx, D.: Open problems in parameterized and exact computation - IWPEC 2006. Technical Report UU-CS-2006-052, Department of Information and Computing Sciences, Utrecht University (2006)

5. Bodlaender, H.L., Drange, P.G., Dregi, M.S., Fomin, F.V., Lokshtanov, D., Pilipczuk, M.: A $c^{k} n$ 5-approximation algorithm for treewidth. SIAM J. Comput. 45(2), 317-378 (2016)

6. Bodlaender, H.L., Fellows, M.R., Thilikos, D.M.: Derivation of algorithms for cutwidth and related graph layout parameters. J. Comput. Syst. Sci. 75(4), 231-244 (2009)

7. Bodlaender, H.L., Gustedt, J., Telle, J.A.: Linear-time register allocation for a fixed number of registers. In: Proceedings of the 9th Annual ACM-SIAM Symposium on Discrete Algorithms, SODA 1998, pp. 574-583. ACM/SIAM (1998)

8. Bodlaender, H.L., Kloks, T.: Efficient and constructive algorithms for the pathwidth and treewidth of graphs. J. Algor. 21(2), 358-402 (1996)

9. Bodlaender, H.L., Thilikos, D.M.: Constructive linear time algorithms for branchwidth. In: Proceedings 24th International Colloquium on Automata, Languages and Programming, ICALP 1997, volume 1256 of Lecture Notes in Computer Science (LNCS), pp. 627-637. Springer (1997)

10. Bodlaender, H.L., Thilikos, D.M.: Computing small search numbers in linear time. In: Proceedings of the 1st International Workshop on Parameterized and Exact Computation, IWPEC 2004, volume 3162 of Lecture Notes in Computer Science (LNCS), pp. 37-48. Springer (2004)

11. Bojanczyk, M., Pilipczuk, M.: Optimizing tree decompositions in MSO. In: Vollmer, H., Vallée, B. (eds.) Proceedings of the 34th Symposium on Theoretical Aspects of Computer Science, STACS 2017, volume 66 of Leibniz International Proceedings in Informatics (LIPIcs), pp. 15:1-15:13 (2017)

12. Feige, U., Hajiaghayi, M.T., Lee, J.R.: Improved approximation algorithms for minimum weight vertex separators. SIAM J. Comput. 38(2), 629-657 (2008)

13. Fürer, M.: Faster computation of path-width. In: Proceedings 27th International Workshop on Combinatorial Algorithms, IWOCA 2016, volume 9843 of Lecture Notes in Computer Science (LNCS), pp. 385-396. Springer (2016)

14. Jeong, J., Kim, E.J., Oum, S.: The "art of trellis decoding" is fixed-parameter tractable. IEEE Trans Inf Theory 63(11), 7178-7205 (2017)

15. Kessler, C.W.: Scheduling expression DAGs for minimal register need. Comput. Lang. 24(1), 33-53 (1998)

16. Lagergren, J.: Efficient parallel algorithms for graphs of bounded tree-width. J. Algor. 20(1), 20-44 (1996)

17. Lagergren, J., Arnborg, S.: Finding minimal forbidden minors using a finite congruence. In: Proceedings of the 18th International Colloquium on Automata, Languages and Programming, ICALP 1991, volume 510 of Lecture Notes in Computer Science (LNCS), pp. 532-543. Springer (1991)

18. Reed, B.A.: Finding approximate separators and computing tree width quickly. In: Proceedings of the 24th Annual ACM Symposium on Theory of Computing, STOC 1992, pp. 221-228. ACM (1992)

19. Robertson, N., Seymour, P.D.: Graph Minors. XIII. The disjoint paths problem. J. Combinat. Theory Ser. B 63(1), 65-110 (1995)

20. Sethi, R.: Complete register allocation problems. SIAM J. Comput. 4(3), 226-248 (1975) 
21. Sethi, R., Ullman, J.D.: The generation of optimal code for arithmetic expressions. J. ACM 17(4), 715-728 (1970)

22. Stanley, R.P. Enumerative Combinatorics, 2nd edn., vol. I. Cambridge University Press, Cambridge (2011)

23. Thilikos, D.M., Serna, M.J., Bodlaender, H.L.: Cutwidth i: a linear time fixed parameter algorithm. J. Algor. 56(1), 1-24 (2005)

24. Thilikos, D.M., Serna, M.J., Bodlaender, H.L.: Cutwidth II: algorithms for partial w-trees of bounded degree. J. Algor. 56(1), 25-49 (2005)

25. Valdes, J., Tarjan, R.E., Lawler, E.L.: The recognition of series-parallel digraphs. SIAM J. Comput. 11(2), 298-313 (1982)

Publisher's Note Springer Nature remains neutral with regard to jurisdictional claims in published maps and institutional affiliations.

\section{Affiliations}

\section{Hans L. Bodlaender ${ }^{1}$ (D) . Lars Jaffke ${ }^{2}$ (D) . Jan Arne Telle ${ }^{2}$}

Hans L. Bodlaender

h.1.bodlaender@uu.nl

Jan Arne Telle

jan.arne.telle@uib.no

1 Utrecht University, Utrecht, The Netherlands

2 University of Bergen, Bergen, Norway 